Mon. Not. R. Astron. Soc. 000, 1-22 (2017) Printed 7 June $2021 \quad$ (MN LATEX style file v2.2)

\title{
The masses and metallicities of stellar haloes reflect galactic merger histories
}

\author{
Richard D'Souza ${ }^{1}{ }^{2},{ }^{\star}$ Eric F. Bell ${ }^{1}$ \\ ${ }^{1}$ University of Michigan, Department of Astronomy, 311 West Hall, 1085 South University Ave., Ann Arbor, MI 48109-1107 \\ ${ }^{2}$ Vatican Observatory, Specola Vaticana, V-00120, Vatican City State
}

Accepted . Received ; in original form

\begin{abstract}
There is increasing observational and theoretical evidence for a correlation between the metallicity and the mass of the stellar halo for galaxies with Milky Way-like stellar masses. Using the Illustris cosmological hydrodynamical simulations, we find that this relationship arises because a single massive massive progenitor contributes the bulk of the mass to the accreted stellar component as well as sets its metallicity. Moreover, in the Illustris simulations, this relationship extends over 3 orders of magnitude in accreted stellar mass for central galaxies. We show that for Milky Way-like mass galaxies, the scatter in accreted metallicity at a fixed accreted stellar mass encodes information about the stellar mass of the dominant accreted progenitor, while the radial density and metallicity gradients of the accreted stellar component encodes information about the time of accretion of the dominant progenitor. We demonstrate that for Milky Way-like mass galaxies, the Illustris simulations predict that the metallicity and the stellar mass of the total accreted stellar component can be reconstructed from aperture measurements of the stellar halo along the minor axis of edge-on disk galaxies. These correlations highlight the potential for observational studies of stellar halos to quantify our understanding of the most dominant events in the growth history of galaxies. We explore the implications of our model for our understanding of the accretion histories of the Milky Way, M31 and NGC 5128. In particular, a relatively late and massive accretion is favoured for M31; additionally, we provide a first estimate of the accreted stellar mass for NGC 5128 .
\end{abstract}

Key words: galaxies: general - galaxies:formation - galaxies: evolution - galaxies: haloes - galaxies: stellar content - galaxies: structure - Galaxy: halo

\section{INTRODUCTION}

The stellar halo of a galaxy encodes information about its accretion history, and has been the raison d'être for the community to pursue the study of the faint surface brightness stellar haloes around galaxies. Today, we have amassed detailed resolved stellar maps of the halo of a handful of nearby galaxies (for example Mouhcine et al. 2010; Ibata et al. 2014; Crnojević et al. 2016). Yet, little progress has been made towards deciphering galactic accretion histories from these detailed observational maps of their stellar haloes. Nevertheless, as the number of observations of the stellar haloes of nearby galaxies have increased, we have begun to appreciate the diversity in the accretion histories of $\mathrm{L}_{*}$ galaxies (Mouhcine et al. 2005; Monachesi et al. 2016; Merritt et al. 2016; Harmsen et al. 2017).

Hints of this diversity were first apparent in compar-

* Contact e-mail:radsouza@umich.edu ing the metallicity of the stellar halo of our own Milky Way (MW) with that of its neighbour, M31 (Mould \& Kristian 1986; Reitzel et al. 1998; Durrell et al. 2001; Ferguson et al. 2002; Durrell et al. 2004). Despite comparable total galactic luminosities and stellar masses, the stellar halo of M31 has a mean metallicity which is nearly an order of magnitude higher than the stellar halo of the MW. Further evidence from neighbouring disk $\mathrm{L}_{*}$ galaxies indicated a range in metallicity in their stellar haloes (Mouhcine et al. 2005). With the exception of the MW, the metallicity of the stellar haloes of these galaxies correlates with the total galaxy luminosity (Mouhcine et al. 2005). As a bright galaxy with an old metal-poor stellar halo, the MW appeared quite rare.

Further evidence of this diversity came in recent measurements of the stellar mass of the halo of nearby disk $\mathrm{L}_{*}$ galaxies (Trujillo \& Fliri 2016; Merritt et al. 2016; Harmsen et al. 2017). Indeed, the masses of most of the stellar haloes of nearby disk galaxies (Merritt et al. 2016; Harmsen et al. 
2017) lie between the extremes charted out by the Milky Way (Bell et al. 2008) and M31 (Ibata et al. 2014).

With the help of early pioneering simulations, the diversity in the metallicity of the stellar haloes was interpreted in terms of differences in their assembly histories (Renda et al. 2005; Font et al. 2006). Renda et al. (2005) showed that galaxies with more extended merging histories possessed stellar haloes with younger and more metal rich stellar populations than the stellar haloes of galaxies with a more abbreviated assembly history (see also Purcell et al. 2008). Moreover, these simulations predicted a relationship between the metallicity and the mass of the stellar halo. Using the particle-tagging simulations of Bullock \& Johnston (2005), Font et al. (2006) also demonstrated a relationship between the metallicity and stellar mass of their accreted haloes of their 11 disk-like galaxies, showing that the relationship mimicked the relationship between the metallicitystellar mass relationship of dwarf galaxies (Gallazzi et al. 2005; Woo et al. 2008) which were destroyed to build up the accreted stellar halo. Based on these models, Robertson et al. (2005) proposed that the diversity in the metallicities of the stellar haloes of nearby galaxies reflected the diversity in the mass of the most massive progenitors accreted by these galaxies. Recently, Deason et al. (2016) using cosmological accretion-only models of a much larger number of MW-mass haloes confirmed these ideas and showed that the most massive progenitor, with $\mathrm{M}_{*} \sim 10^{8}-10^{10} M_{\odot}$, contributes the bulk of the accreted stellar material and drives the correlation between the metallicity and the mass of the stellar halo.

Recently, Harmsen et al. (2017), using the GHOSTS (Galaxy Halos, Outer disks, Substructure, Thick disks, and Star clusters) survey (Radburn-Smith et al. 2011; Monachesi et al. 2016), demonstrated for the first time observationally a relationship between the metallicity of halo stars (measured at $30 \mathrm{kpc}$ along the minor axis) and the mass of the stellar halo of 6 nearby MW-size edge-on disk galaxies (plus the MW and M31). Given the predicted variations in the accretion histories of MW-like galaxies, the existence of such a relationship between the metallicity and the mass of the stellar halo of nearby galaxies is truly remarkable, and suggests that these Hubble Space Telescope (HST) observations along the minor axis of these nearby MW-peers are able to probe the characteristics of the most massive progenitor accreted by these galaxies. This opens the possibility of empirically exploring the relationship between the most massive merger/accretion partner and galactic properties (e.g., Bell et al. 2017) — a potentially crucial insight into how merging and accretion affect galaxies.

In light of this new observational evidence and additional constraints on the galaxy metallicity- stellar mass relationship for dwarf galaxies (Kirby et al. 2013), it is appropriate to revisit the modelling of the relationship between the metallicity and the stellar halo mass for a range of galaxy types and sizes, in order to understand how informative this relationship is about the accretion history of the galaxy, especially in terms of the characteristics of the most massive accreted progenitors. The availability of large cosmological hydrodynamical simulations (e.g. Illustris and EAGLE; Vogelsberger et al. 2014b; Schaye et al. 2015) allows us to study the correlation between the metallicity and mass of the stellar halo of galaxies for a broader range in DM halo masses and accretion histories, while the spatial resolution of these simulations allows us to connect these quantities more directly to observable ones.

In this study, we use the GHOSTS HST observations as a template for possible observable quantities. The GHOSTS survey targeted nearby edge-on disk galaxies with pencil beam observations, where stellar halos were probed using pointings along the minor axis (or axes) with galactocentric distances $5<d_{\text {minor axis }}<80 \mathrm{kpc}$, and more typically $10<d_{\text {minor axis }}<80 \mathrm{kpc}$. Additionally, a power-law density profile and a metallicity gradient of the stellar halo can also be quantified at this distance. Comparing these observations with accretion-only models, Harmsen et al. (2017) concluded that the halo stars probed at these distances were predominately of accreted origin.

However, hydrodynamical simulations also predict the presence of in situ stars (born within the galaxy and not accreted) at large galacto-centric distances (Zolotov et al. 2009; Font et al. 2011; Pillepich et al. 2015), commonly called as the 'in situ' stellar halo. Although quantitative predictions of the amount of contribution of in situ stars to the stellar halo are highly dependent on numerical (e.g., initial conditions, accretion history) and modelling (e.g., prescriptions for star formation and the treatment of the multiphase interstellar medium) techniques employed in the simulations (Zolotov et al. 2009; Cooper et al. 2015), there is a consensus within the community that the stellar halo beyond $30 \mathrm{kpc}$ is dominated by the accreted stellar component (Cooper et al. 2015). The Illustris simulations predict a relatively large fraction of in situ stars at large galacto-centric distances beyond $30 \mathrm{kpc}$ (Pillepich et al. 2014), even along the minor axis. These predictions appear incompatible with present GHOSTS observations (Harmsen et al. 2017, see also section 2.1).

In this work, we instead focus on the accreted stellar component of hydrodynamical simulations. These accreted stars are tidally torn from accreted dwarf galaxies. The ingredients that are vital to building up the accreted stellar component of a galaxy are relatively realistic and simple by design. Consequently, any model that has $i$ ) a realistic mass function of galaxies, ii) reasonable merger histories, and iii) accurately modelled potential wells will produce realistic accreted halos. These requirements are satisfied in present day hydrodynamical simulations. Hence, the accreted stellar component is fairly robustly modelled in the present set of hydrodynamical simulations. On the other, because the in situ stellar component is highly dependent on poorly constrained recipes for star formation and stellar feedback in the outer lower density regions of a galaxy, they are extremely sensitive to a number of modelling dependencies and vary considerably from simulation-to-simulation.

In this work, we also focus on the total accreted stellar component, as opposed to only the accreted stellar material beyond a certain galacto-centric radius. We adopt this approach in order to examine those physical quantities that are most informative about the accretion history of the galaxy. Yet, given that much of the accreted material is expected to have settled into the centre of the galaxy where it is observationally difficult to access, even for minor axis resolved stellar populations studies, we will explore also how minor axis properties at larger radii relate to the total accreted quantities. Furthermore, for MW-like galaxies, we assume 
that the stellar halo probed at $30 \mathrm{kpc}$ along the minor axis (GHOSTS HST observations) is predominately of accreted origin.

In Section 2, we review the suitability of using the accreted stellar component of the Illustris galaxies to study the stellar haloes of MW-mass galaxies. In Section 3, we demonstrate that the bulk of the accreted stellar component is built up by the most massive accreted progenitor, and that it drives the accreted metallicity-stellar mass relationship. In Section 4, we show how the scatter in the accreted metallicity relationship is informative about the fraction of accreted stellar mass contributed by the dominant progenitor. In Section 5, we explore how the physical properties (e.g, density or metallicity gradients) of the accreted stellar component of MW-like mass galaxies encodes information about the time of accretion of the dominant progenitor. In Section 6, we demonstrate how it is possible to reconstruct the mass and metallicity of the total accreted stellar component from observable "aperture" measurements. Finally, in Section 7, we discuss our results and their implications for the MW, M31 and Cen A.

\section{ILLUSTRIS SIMULATIONS}

We use the public release of the Illustris suite of simulations (which are described in detail in Vogelsberger et al. 2014b; Genel et al. 2014; Vogelsberger et al. 2014a). These are large cosmological hydro-dynamical simulations (with a periodic box of $106.5 \mathrm{Mpc}$ on a side) run with the AREPO code (Springel 2010) and include key physical processes that are believed to be relevant for galaxy formation (Vogelsberger et al. 2013) including gas cooling with radiative self-shielding corrections, star formation, energetic feedback from growing SMBHs and exploding supernovae, stellar evolution with associated chemical enrichment and stellar mass loss, and radiation proximity effects for AGNs. The Illustris simulations produce a morphologically diverse galaxy population, reproducing median morphology trends with stellar mass, SFR and compactness (Snyder et al. 2015). The details of the public release are found in Nelson et al. (2015).

We use Illustris-1, the highest resolution run, which follows the evolution of dark matter (DM), cosmic gas, stars and super massive black holes from a starting redshift of $z=127$ to $z=0$ with cosmological parameters consistent with WMAP-9 results. This run has a DM mass resolution of $\mathrm{m}_{\mathrm{DM}}=6.26 \times 10^{6} M_{\odot}$ and a resolution of $\mathrm{m}_{\text {baryon }}=1.26 \times 10^{6} M_{\odot}$ for the baryonic component. The gravitational softening lengths are 1.4 and $0.7 \mathrm{kpc}$ for the DM and baryonic particles respectively at $z=0$. Haloes, subhaloes and their basic properties have been identified using the Friends-of-Friends (FoF) and SUBFIND algorithms (Davis et al. 1985; Springel et al. 2001; Dolag et al. 2009). We use the merger trees as identified by the SuBLink code (Rodriguez-Gomez et al. 2015).

In this work, we use a Hubble constant of $\mathrm{H}_{0}=72 \mathrm{~km} \mathrm{~s}^{-1} \mathrm{Mpc}^{-1}$. For the virial mass of a particular galaxy, we use the total mass of the group enclosed in a sphere whose density is 200 times the critical density of the Universe (contained in Group_M_Crit200). For the stellar mass of a particular galaxy, we use the stellar mass of all the stellar particles bound to this subhalo (contained in SubhaloMassType).

We use the metallicity values (all elements above $\mathrm{He}$ ) of the stellar particles as found in the snapshots and the halo catalogues. These metallicity values are inherited from the gas cell which get converted into stars at the time of their birth. The metallicity values found in the Illustris simulations are a product of their choice of stellar evolution and metal enrichment. Furthermore, no metallicity floor has been imposed to the output data, so that the metallicities of a small fraction of gas and star elements adopt minuscule, unrealistic values. We impose a lower limit to the stellar metallicities in this work $([\mathrm{M} / \mathrm{H}] \sim-5.4)$. The median fraction of stellar particles affected is $0.1 \%$, with an upper limit of $0.2 \%$ for smaller galaxies. We find that these stellar particles with unrealistic metallicities have a minimal effect on the median accreted stellar metallicity of galaxies. We choose to retain these stellar particles with a lower limit in stellar metallicities, so as not to bias our estimates of the accreted stellar mass.

The Illustris metallicity model is dependent upon the sub-grid "physics" models (e.g., stellar evolution, chemical enrichment of SN Ia/II and AGB stars, as well as AGN feedback and stellar winds) implemented in the simulation. Given these uncertainties, it is imperative that we compare the metallicity of the Illustris galaxies as a function of their stellar mass (both overall normalisation and shape of the relationship) with the relevant observational constraints available in the literature. We do so in Figure 1: the stellar metallicity of SDSS galaxies (Gallazzi et al. 2005) and of dwarf satellites of the Milky Way and M31 (Kirby et al. 2013). While Gallazzi et al. 2005 measure metallicities using broad spectral features, dominated by $\mathrm{Mg}$ and $\mathrm{Fe}$ in the integrated light of galaxies, Kirby et al. 2013 measure [Fe/H] from iron absorption lines in individual stars. For the purpose of this paper, we treat them equivalently as tracing predominately $[\mathrm{Fe} / \mathrm{H}]$. On the other hand, the Illustris model traces the total stellar metallicity $[\mathrm{M} / \mathrm{H}]$ (all elements above $\mathrm{He}$ ). In this work, we lower the metallicities of the Illustris galaxies by 0.3 dex to convert it to a $[\mathrm{Fe} / \mathrm{H}]$ system and adjust for any limitations in the Illustris chemical evolution model. Doing so provides a a good match between the overall normalisation of the metallicity of the Illustris galaxies and the observational constraints.

The mean metallicity of the Illustris galaxies increases with stellar mass in reasonable accord with observations, although the exact shape of the Illustris stellar massmetallicity mass relationship appears to differ somewhat from the observations. In general, the mass-metallicity relationship of SDSS $\mathrm{L}_{*}$ galaxies is steeper than that found in the Illustris simulations, while the mass-metallicity relationship of dwarf galaxies is shallower than found in the simulations. The Illustris predictions are a direct consequence of the choice of the Illustris stellar evolution model as well as the recipes of galactic winds and AGN feedback employed in the simulation. The slope of the mass-metallicity relationship of galaxies will have a direct impact on the accreted stellar metallicities derived later.

The Illustris simulations shows weak evolution of the stellar metallicity-stellar mass relationship with redshift with redshift (See Figure 2). This behaviour is not inevitable: the simulations of Ma et al. (2016) exhibit a strong 
time evolution in the stellar metallicity-stellar mass relationship. At a fixed stellar mass, they predict a strong evolution in stellar metallicity of nearly 0.5 dex from $z \sim 3$ to $z \sim 0 .^{1}$

There are few observational constraints on the evolution of the stellar metallicity-stellar mass relationship with redshift. Although gas-phase metallicities have been measured to redshift $z \sim 2$ using gas-phase oxygen and nitrogen abundances of the interstellar medium, future estimates of the stellar metallicities of high redshift galaxies will provide crucial data for our present understanding of chemical evolution models which have not been reliably assessed outside the local Universe. Determining stellar metallicities for galaxies at high redshift is a difficult task and requires deep spectroscopy in order to trace the stellar continuum and the strength of key absorption features chiefly sensitive to age and metallicity. A few studies have attempted to push to higher redshift $(z \sim 2)$ by using rest-frame UV absorption features (Rix et al. 2004; Halliday et al. 2008; Sommariva et al. 2012). Gallazzi et al. (2014) using deep multi-object optical spectroscopy found very little evolution in the stellar metallicity between $z \sim 0.7$ and $z \sim 0.1$ (a difference of 0.13 dex). Based on the limited observational evidence available so far, we conclude that the Illustris chemical evolution model is more consistent with the observations than the simulations of Ma et al. (2016).

Given the overall reasonable agreement between Illustris and the observed metallicities of galaxies, and cognizant of its uncertainties, we proceed to analyze the properties of Illustris central galaxies. We select 4644 central galaxies at $z=0$ with DM subhalo masses $\log _{10} \mathrm{M}_{\text {halo }} / \mathrm{h} \geqslant 11.5$. These central galaxies are those that have the most massive SuBFInD subhaloes within a FOF group. We ensure that each galaxy is resolved by more than 3500 stellar particles per halo. These galaxies span a range in accretion histories and galaxy morphologies.

In this work, we identify a stellar particle as in situ if the galaxy in which it formed lies along the main progenitor branch of the galaxy (SUBLink merger trees RodriguezGomez et al. 2015) in which it is currently found. The other stellar particles found in the subhalo of the main galaxy are tagged as "accreted". We note that our definition of "accreted" stars includes those stellar particles formed from gas stripped from satellite galaxies and are eventually bound to the main subhalo of the host galaxy. Our definition of in situ versus accreted stars is consistent with RodriguezGomez et al. (2016). Our classification of stars formed from gas stripped from satellite galaxies is closer to Pillepich et al. (2015) than Cooper et al. (2015). It is worth noting that in simulations using the AREPO code like Illustris, it is difficult to trace the origin of the gas particle responsible for the creation of a stellar particle. Hence, it is difficult to distinguish

1 The simulations of Ma et al. (2016) attribute the evolution in stellar metallicity with time at a fixed stellar mass to their different rates of star formation over cosmic time. In their simulations, the fraction of stellar material to the total number of baryons (including the halo gas) increases with time. In other words, the suppression of star formation at high redshift leads to a decrease in the amount of metals produced in galaxies. In contrast, in other simulations galaxies tend to form a large fraction of their stars at higher redshift.

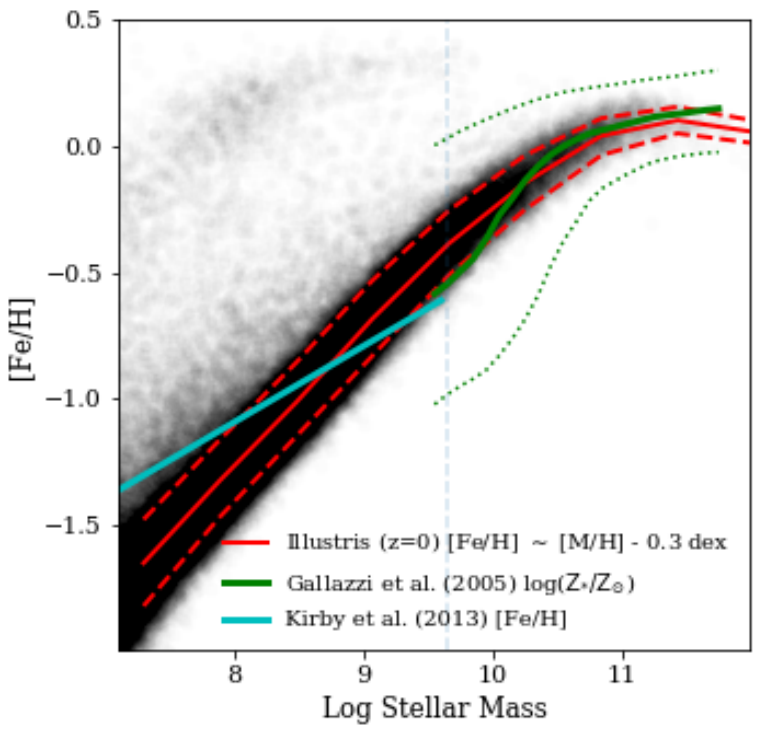

Figure 1. The mass-weighted mean metallicity of Illustris central galaxies as a function of the galaxy stellar mass. The red solid line and dashed lines indicate the median and the 16/84 percentile of the distribution. The metallicity of the Illustris galaxies is lowered by 0.3 dex to convert it to $[\mathrm{Fe} / \mathrm{H}]$. Observational constraints: The solid green line indicates the metallicity of SDSS galaxies as estimated by Gallazzi et al. (2005). The dotted green lines indicate the 16 th and 84 th percentile of the distribution. The solid cyan line are observational constraints of dwarf galaxies from Kirby et al. (2013). The Illustris mass-metallicity relationship is reasonably consistent and reproduces the broad contours of the observational data.

stars formed from 'stripped gas' (from satellites) as opposed to stars formed from 'smoothly accreted gas'. Indeed stars formed from gas stripped from satellites after infall into the main galaxy often share some of the properties of stars born before infall. The definitions we adopt in this paper, especially with regards to stars formed from gas stripped from satellites, are consistent with our overall aim of studying the accretion history of the galaxy.

We define the accreted stellar mass of a galaxy as the mass of stars born external to the main progenitor branch of the galaxy $\left(\mathrm{M}_{\mathrm{acc}}\right)$. For a few star-forming galaxies, the subhalo finder algorithm SuBFIND sometimes identifies star forming regions as separate subhaloes. We consider any such groupings of stellar particles identified by the SUBFIND algorithm as separate subhaloes without any associated DM as part of the in-situ stellar component of the galaxy.

For each galaxy, we identify the 'dominant' progenitor which contributes the largest amount of accreted stellar material to the galaxy. We identify the mass of the dominant progenitor $\left(\mathrm{M}_{\mathrm{dom}}\right)$ as the maximum mass of the progenitor satellite before it becomes accreted onto the main progenitor branch of the galaxy (see SuBLink merger trees above). We also quantify the fraction of accreted stellar material which was contributed by the most dominant progenitor $\left(f_{\text {Dom }}\right)$. We note that due to stripping due to tidal effects, in some cases, $\mathrm{M}_{\text {Dom }}$ may be larger than $\mathrm{f}_{\text {Dom }} \times \mathrm{M}_{\mathrm{Acc}}$. Using the SuBLINK merger trees, we can also identify the time of accretion of the progenitor by the main galaxy. This corresponds to 


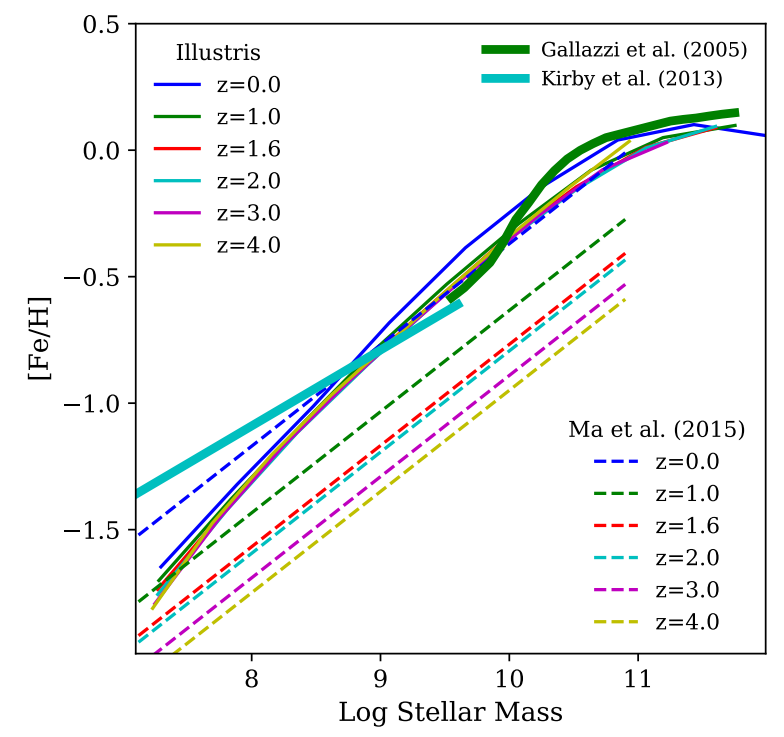

Figure 2. The lack of evolution in the metallicity-stellar mass relationship of Illustris galaxies from redshift $\mathrm{z}=4$ to the present day universe (solid thin lines). We also compare this with evolution of galaxy metallicity-stellar mass relationship in the simulations of Ma et al. 2015 (dashed thin lines). For comparison, we also show the observational results of the metallicity-stellar mass relationship of local dwarfs of Kirby et al. (2013) and of SDSS galaxies from Gallazzi et al. (2005).

the time the SuBFIND algorithm can no longer identify the stellar particles associated with a particular progenitor as a separate entity but bound to the main galaxy. Note that in this work, $t$ refers to cosmic time, or the age of the Universe. Hence, $\mathrm{t}=0$ corresponds to the start of the simulation, or the Big Bang.

We characterise the first moment of the accreted stellar metallicity distribution function (MDF) in terms of the mass-weighted mean and the median metallicity. We find that the mass-weighted mean metallicity of the accreted stellar component is at the most 0.15 dex higher than the median metallicity. For a few isolated cases $(<10)$, very recent and large accretion events ('giant streams') bias the massweighted metallicity to much higher values. For the purposes of this paper, we only consider the median metallicity of the accreted stellar component. We do not identify and remove recent accretion events that in observations may appear as 'Sagittarius'-like streams or objects. Not only would removal of such accretion events be explicitly resolution-dependent, but also GHOSTS-like observational studies often cannot recognize and do not remove such substructure.

To compare our results with the GHOSTS observations of neighbouring edge-on spiral galaxies (Harmsen et al. 2017), we consider the accreted stellar particles in a narrow wedge along the minor axis. For minor axis properties, we orient each galaxy along its principal axis and estimate the metallicity of the accreted stellar component in two projected wedges of 30 degrees width along the minor axis between a galacto-centric distance of 25 and $45 \mathrm{kpc}$. To increase the number of particles and smooth out sudden variations due to the presence of substructure, we include parti- cles on both axes located within the diametrically opposed wedge.

We also characterise the (observationally-inaccessible) size of the accreted stellar component in terms of $3 \mathrm{D}$ radial distance which contains $50 \%$ of its stellar mass $\left(R_{50 \text { Acc }}\right)$. We estimate the projected radial density profile of the accreted stellar component along the minor axis, and estimate its power-law slope $\left(\Gamma_{\text {Min Acc }}\right)$. Finally, we also estimate the projected accreted stellar metallicity gradient along the minor axis.

\subsection{Comparison of the Illustris stellar halos with observations}

We now turn our attention to comparing how well the stellar haloes of the Illustris galaxies matches the observational data. We first examine the bulk properties of the stellar haloes of the GHOSTS galaxies and compare them with the accreted stellar component of the Illustris galaxies. In Fig. 3 , we show the estimated $[\mathrm{Fe} / \mathrm{H}]$ at $30 \mathrm{kpc}$ along the minor axis of the 6 GHOSTS galaxies (Monachesi et al. 2016), along with the Milky Way and M31, as a function of their estimated stellar halo masses measured in an elliptical aperture measured between 10 and $40 \mathrm{kpc}$ along the semi-minor axis (as in Figure 12(b) of Harmsen et al. 2017).

We compare these observations with the predictions of the accreted stellar component from the Illustris simulations. We select GHOSTS-like galaxies from the Illustris simulation by choosing low-concentration galaxies in a narrow stellar mass range $\left(10.5<\log \mathrm{M}_{*}<11.0, \mathrm{R}_{90} / \mathrm{R}_{50}<2.4\right)$. We estimate the median accreted stellar metallicity $[\mathrm{Fe} / \mathrm{H}]$ in a narrow wedge along the minor axis for these GHOSTSlike Illustris galaxies (shaded points). We also estimate an aperture accreted stellar mass between 15 and $50 \mathrm{kpc}$ along the semi-minor axis, corresponding approximately to an elliptical aperture measured between 10 and $40 \mathrm{kpc}$ along the minor axis.

Recall that the estimated $[\mathrm{Fe} / \mathrm{H}]$ scaling that we have adopted for Illustris $([\mathrm{M} / \mathrm{H}]-0.3 \mathrm{dex})$ gives a good match to the galaxy metallicity-stellar mass relationship (Fig. 1). With such an adjustment, the agreement between the Illustris predictions of the outer accreted stellar component and the observational constraints is quite remarkable. Furthermore, the distribution of the predicted aperture accreted stellar mass (top panel of Figure 3) matches the GHOSTS data well. In contrast, the distribution function of the aperture stellar halo masses (in situ + accreted; in green) of Illustris GHOSTS-like galaxies dramatically over-predicts the amount of stellar mass at large radii in the GHOSTS sample of galaxies.

Having demonstrated overall agreement with the bulk properties of the stellar halo of the GHOSTS galaxies and the accreted stellar component of the Illustris simulations, we proceed to a more stringent comparison of the surface mass density profile of the GHOSTS galaxies along the minor axis, with the profiles predicted from the Illustris simulations. In Figure 4, we compare the estimated surface mass density profiles of the GHOSTS galaxies with the profile of Illustris GHOSTS-like galaxies (selected above). We assume a constant $\mathrm{M} / \mathrm{L}$ ratio in the $\mathrm{V}$-band of 2.5. We also include the observational constraints of the profiles of M31 along the minor axis (Irwin et al. 2005; Gilbert et al. 2012) 


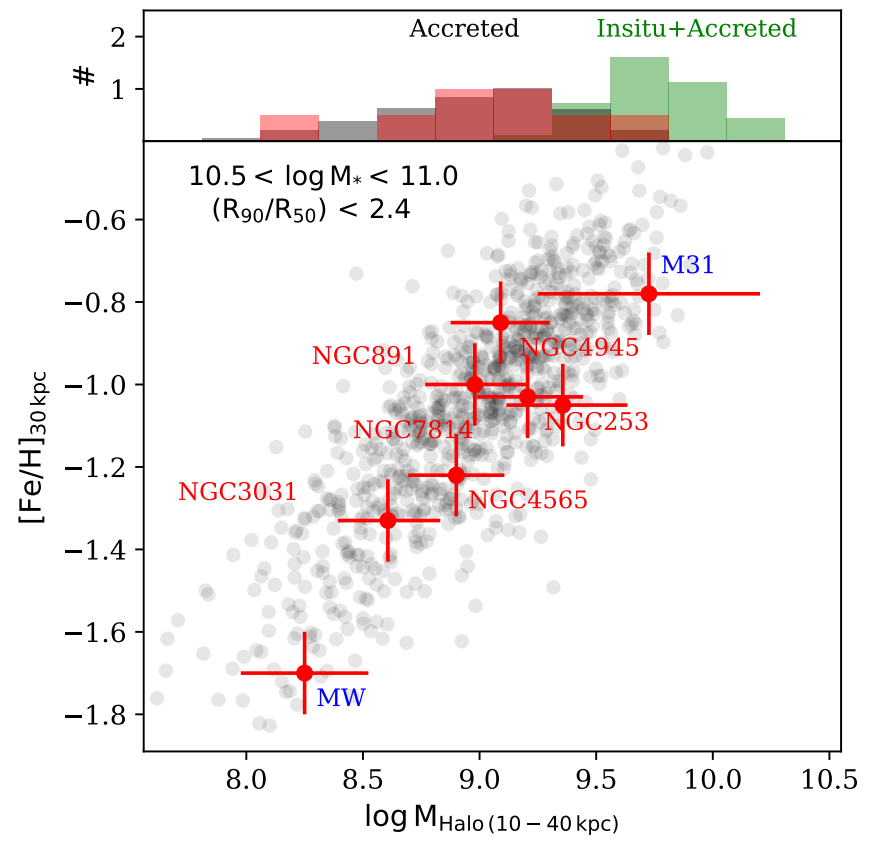

Figure 3. Bottom Panel: Accreted metallicity of the 6 GHOSTS galaxies (Harmsen et al. 2016) in addition to the Milky Way and M31 (plotted as a function of their 'aperture' stellar halo mass measured between 10 and $40 \mathrm{kpc}$ ). Measurements of Milky Way and M31 are also taken from Harmsen et al. who used data from Bland-Hawthorn \& Gerhard 2016, Xue et al. 2015, Ibata et al. 2014, Gilbert et al 2014. The grey points indicated the accreted stellar metallicity estimated in a wedge along the minor axis for Illustris GHOSTS-like galaxies $\left(10.5<\log \mathrm{M}_{*}<11.0\right.$, $\left.\mathrm{R}_{90} / \mathrm{R}_{50}<2.4\right)$ as a function of the accreted stellar mass measured in an aperture between 10 and $40 \mathrm{kpc}$. Top Panel: We compare the distribution function of the aperture accreted stellar masses of Illustris GHOSTS-like galaxies (grey) with the aperture stellar halo masses of the GHOSTS data + MW + M31 (red) measured between 10 and $40 \mathrm{kpc}$, finding that they are consistent with the accreted stellar component only. In contrast, the distribution function of the aperture stellar halo masses (in situ + accreted) of Illustris GHOSTS-like galaxies is not consistent with the observational data.

in our comparison. We find that the median surface density profile (along with the spread) of the accreted stellar component of Illustris GHOSTS-like galaxies matches the observational constraints quite well, especially between 10 and $50 \mathrm{kpc}$ along the minor axis. On the other hand, the median surface density profile of the total (in situ + accreted) is comparatively larger than the observational constraints. Note that owing to the mass resolution of the Illustris simulations, the surface mass density profiles do not extend lower than $\log _{10} \Sigma_{*} \sim 4.5 \mathrm{M}_{\odot} / \mathrm{kpc}^{2}$. This implies that the Illustris simulation over-predicts the in situ component along the minor axis. It also validates the claim of Harmsen et al. (2017) that the minor axis profiles of GHOSTS galaxies around $30 \mathrm{kpc}$ are predominately of accreted origin.

The agreement between the Illustris accreted halo and observed halos at large radius is encouraging and supports the idea that exploring the accreted stellar component in Illustris could help build intuition about how to interpret the observed stellar halos. Accordingly, we now turn to a

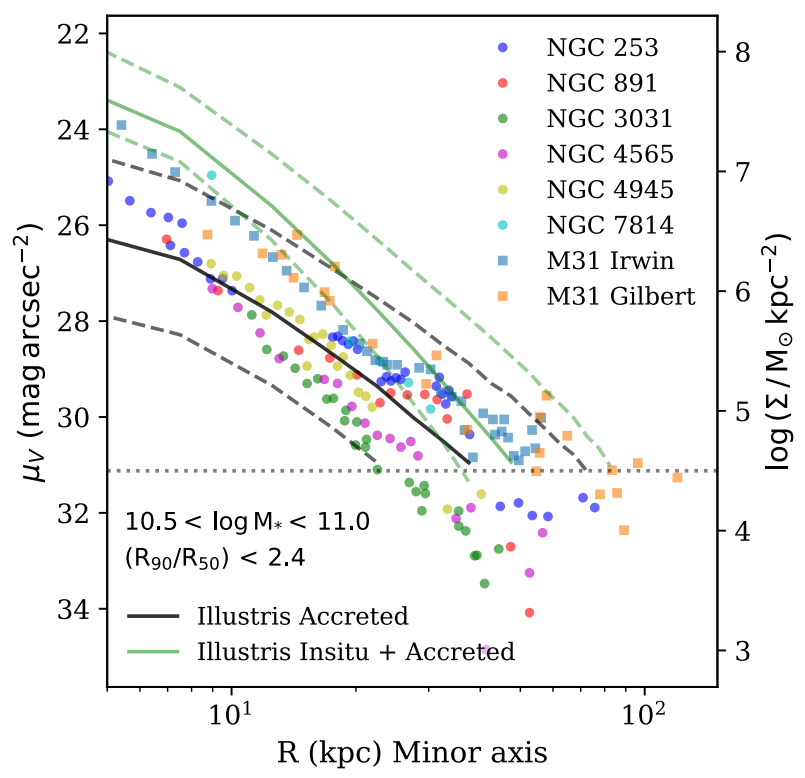

Figure 4. Surface brightness profiles along the minor axis of the 6 GHOSTS galaxies in the V-band from Harmsen et al. 2017. We also add the surface brightness profiles of M31 along the minor axis in the I-band from Irwin et al. 2005 and Gilbert et al. 2012. Assuming a M/L ratio of 2.5 in the V-band, we also plot a surface mass density profile of the total (green) and the accreted stellar component (black) along the minor axis for Illustris GHOSTS-like galaxies $\left(10.5<\log \mathrm{M}_{*}<11.0, \mathrm{R}_{90} / \mathrm{R}_{50}<2.4\right)$. The dashed lines indicate the 16 and 84 percentile of the radial distributions. Owing to the resolution of Illustris, the surface mass density profiles do not extend lower than $\log _{10} \Sigma_{*} \sim 4.5$ (indicated by the horizontal dotted line). The surface mass density profiles of galaxies along the minor axis agree well with only the accreted stellar component beyond a radius of $15 \mathrm{kpc}$. Inclusion of the in situ stellar component over-predicts the surface mass density profiles.

more detailed and systematic study of the Illustris accreted metallicity-stellar mass relationship.

\section{ACCRETED METALLICITY-STELLAR MASS RELATIONSHIP}

We now explore the accreted metallicity-stellar mass relationship for the central galaxies in the Illustris simulations. In Figure 5, we plot the median metallicity of the total accreted stellar component of the Illustris galaxies as a function of the accreted stellar mass colour-coded by the DM halo mass of the galaxy $\left(11.0<\log _{10} \mathrm{M}_{\mathrm{DM}}<14.0\right)$. Illustris shows a tight relationship between the metallicity and the stellar mass of the accreted component extending over three orders of magnitude in accreted stellar mass.

The accreted metallicity-stellar mass relationship follows closely the Illustris median metallicity-stellar mass relationship for galaxies, with the accreted stellar metallicity being on an average 0.3 dex lower than the total median metallicity (we discuss the colour-coding by dark matter halo mass later in $\S 5$ ). At higher accreted stellar mass $\left(\log \mathrm{M}_{\mathrm{acc}} \geqslant 11.0\right)$, the relationship between the metallicity and the accreted stellar mass tapers off. Since the shape 


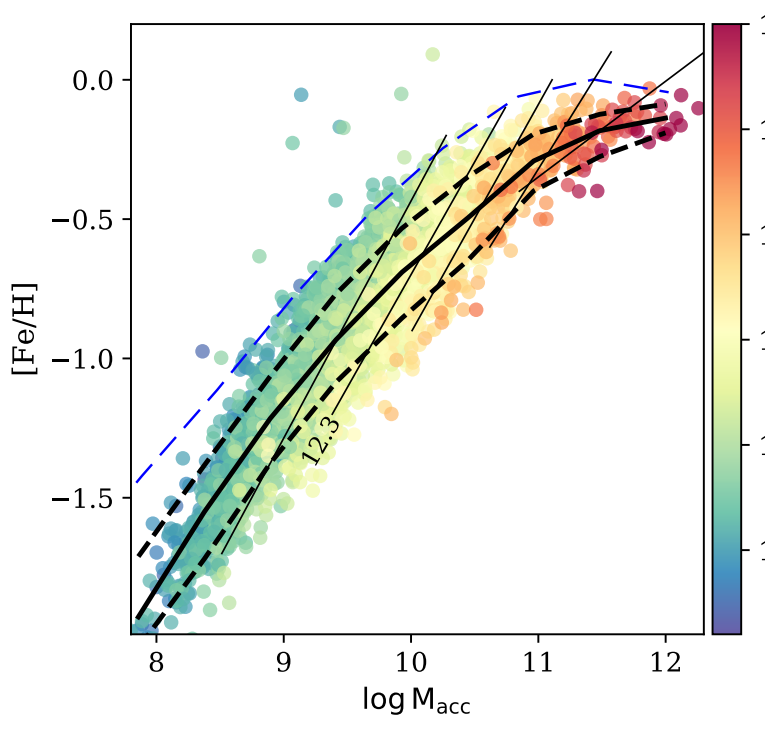

Figure 5. The Illustris accreted metallicity-stellar mass relationship: the metallicity of the accreted component is plotted as a function of the accreted stellar mass of the Illustris galaxies. The black solid and dashed lines indicate the median and the 16/84th percentile of the distribution. The galaxies are coloured by their halo mass. The thin black lines show tracks of similar halo mass (at $\log _{10} \mathrm{M}_{\mathrm{DM}}=11.9,12.3,12.7,13.1 \& 13.5$; discussed more in $\S 5$ ). These contour-like solid lines are fitted to narrow ranges in $\log _{10} \mathrm{M}_{\mathrm{DM}}$. We overplot the median metallicity-stellar mass relationship (blue dashed lines) of galaxies in the Illustris simulations.

of the accreted metallicity-stellar mass relationship follows closely the shape of the metallicity-stellar mass relationship, it suggests that the former is being driven by the accreted satellites following the galaxy metallicity-stellar mass relationship.

In fact, the overall accreted metallicity-stellar mass relationship is being driven by the most massive accreted ("dominant") progenitor of the galaxy, which contributed the bulk of the accreted stellar material of the galaxy. We demonstrate this in Figure 6 by plotting the ratio of the accreted stellar mass to the stellar mass of the dominant progenitor as a function of the accreted stellar mass. The median of this ratio is between 1 and 2 . In contrast, the ratio of the accreted stellar mass to the stellar mass of the second most massive progenitor is greater than 4 . This suggests that the dominant accreted progenitor is on average twice as massive as the second most massive accreted progenitor, and that the overall accreted stellar mass is dominated by the most massive progenitor.

The scatter in the ratio of the the accreted stellar mass to the stellar mass of the dominant progenitor (noted by the 5 th and 95th percentile of the distribution) as a function of the accreted stellar mass indicates that a small fraction of galaxies are built up through small yet significant mergers.

We also plot the difference in metallicity between the accreted stellar component and the dominant progenitor as a function of accreted stellar mass. This difference is nearly

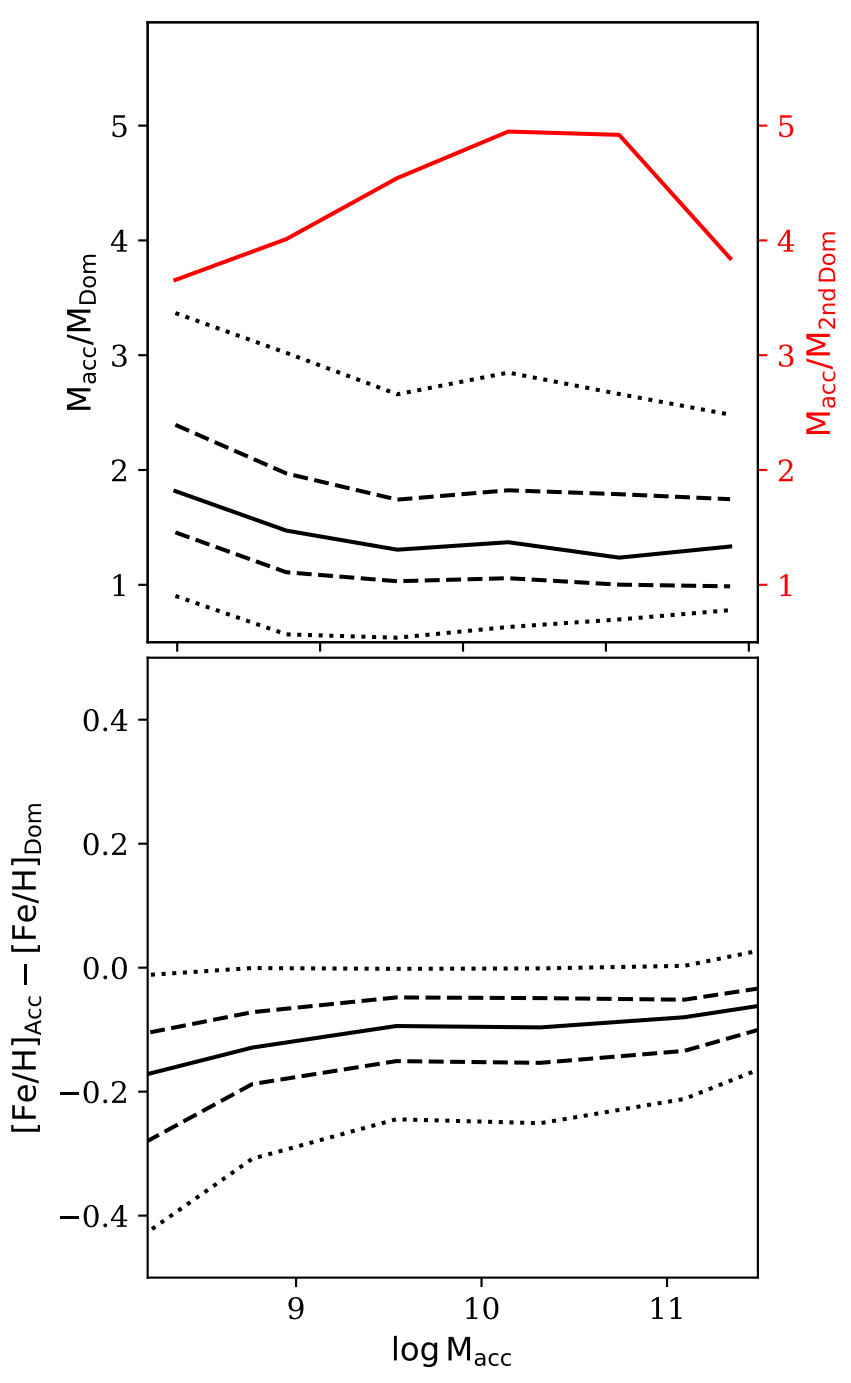

Figure 6. Top panel: The ratio of the accreted stellar mass to the stellar mass of the most massive accreted ("dominant") progenitor as a function of accreted stellar mass. The black solid line indicates the median of the distribution. The dashed lines indicate the 25 th and 75 th percentiles. The dotted lines indicate the 5 th and 95 th percentiles. The red solid line indicates the median of the ratio of the accreted stellar mass to the stellar mass of the second most massive progenitor. The bulk of the accreted stellar component is contributed by the dominant progenitor. Bottom panel: The difference between the median metallicity of the accreted stellar component and the dominant progenitor as a function of accreted stellar mass and halo mass.

constant with accreted stellar mass and is around 0.1 dex in the expected sense that the dominant satellite is typically slightly more metal rich than the overall accreted halo. The maximum scatter in the relationship is around $0.2 \mathrm{dex}$. The low amount of scatter indicates that the metallicity of the dominant progenitor sets the accreted stellar metallicity of the galaxy and drives the general accreted metallicity-stellar mass relationship. 


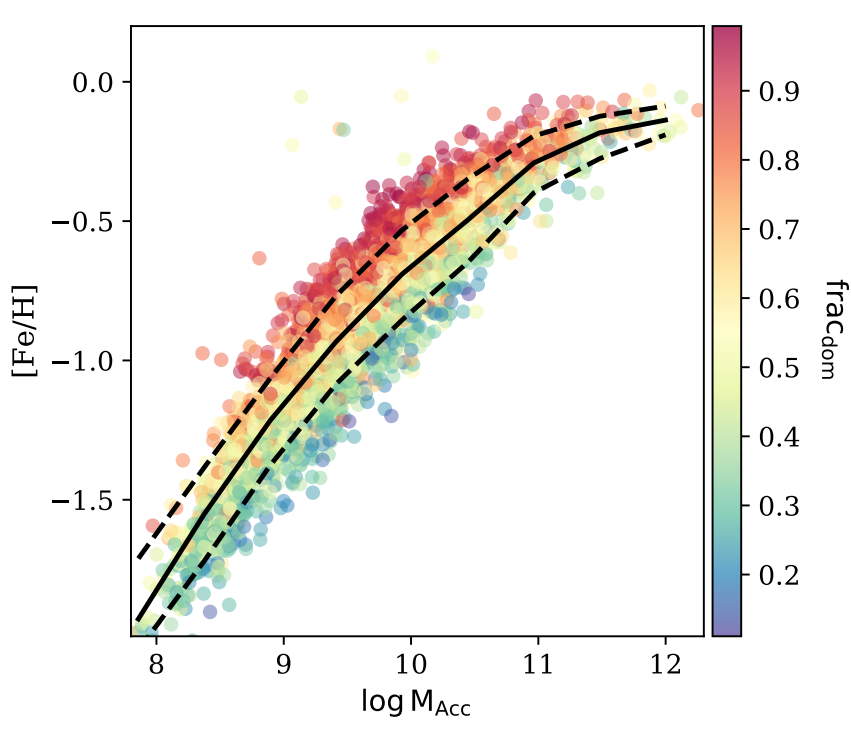

Figure 7. Accreted metallicity-stellar mass relationship colour coded by the fraction of accreted stellar mass (frac ${ }_{\text {Dom }}$ ) contributed by the dominant progenitor. The scatter in the accreted metallicity-stellar mass relationship encodes information about frac $_{\text {Dom }}$.

\section{SCATTER IN THE ACCRETED METALLICITY-STELLAR MASS RELATIONSHIP AT FIXED ACCRETED STELLAR MASS}

We now turn our attention to understand what drives the scatter in the Illustris accreted-stellar mass relationship and how informative it is about the accretion history of a galaxy. As seen in Figure 5, there is 0.2 dex scatter at a given accreted stellar mass in the Illustris accreted metallicity-stellar mass relationship. The scatter at a fixed accreted stellar mass reflects the different possible accretion histories which can build up an accreted stellar component of that mass.

We parametrize the accretion history of a galaxy in terms of the fraction of accreted stellar material contributed by the dominant progenitor frac Dom. This allows us to study how the accreted stellar metallicity varies as a function of the most massive accretion event of the galaxy. In Figure 7, we consider the accreted metallicity-stellar mass relationship

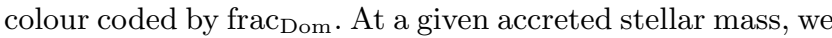
find that galaxies with a higher accreted stellar metallicity have their accreted haloes built up predominately by a single merger event, while galaxies with a lower accreted stellar metallicity have their haloes built up through a number of accreted satellites.

While Fig. 7 shows that high metallicity halos for a given accreted mass tend to have a higher frac ${ }_{D o m}$, it is interesting to consider more closely the contribution of the dominant progenitor to the metallicity at a fixed accreted stellar mass $\left(10.3<\log \mathrm{M}_{\mathrm{acc}}<10.4\right)$ in Figure 8. At higher frac Dom $_{\text {, the metallicity of the accreted stellar compo- }}$ nent approaches the metallicity of the dominant progenitor,

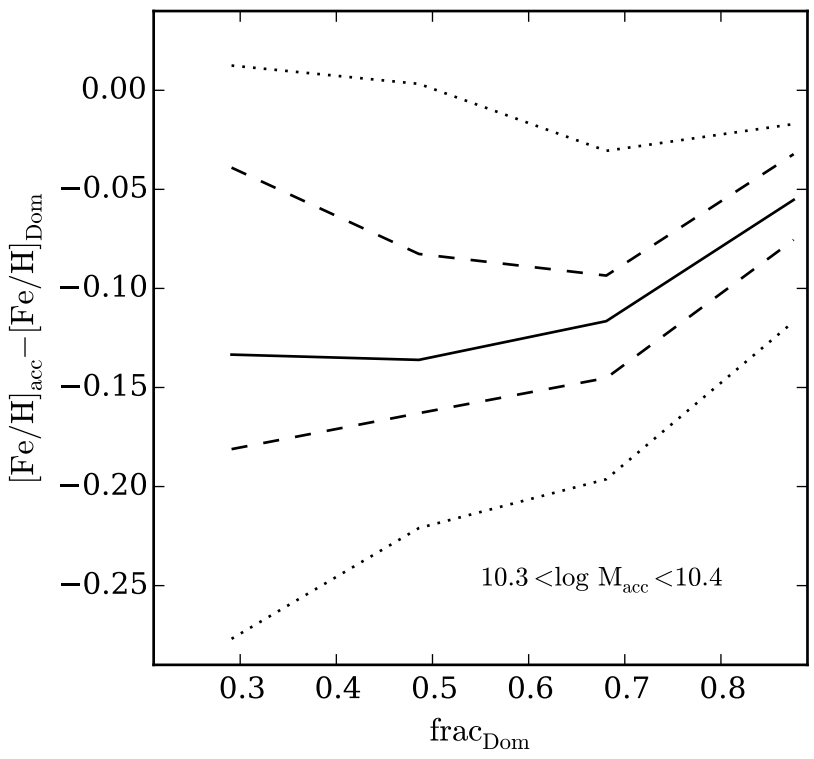

Figure 8. The difference in accreted stellar metallicity and the metallicity of the dominant progenitor as a function of frac Dom for galaxies at a fixed accreted stellar mass $\left(10.3<\log \mathrm{M}_{\mathrm{acc}}<\right.$ 10.4). The dashed lines indicate the 25 th and 75 th percentiles. The dotted lines indicate the 5th and 95th percentiles. At a fixed accreted stellar mass, the spread in accreted metallicity is driven by the most dominant progenitor.

with a scatter considerably smaller than 0.1 dex. At lower frac $_{\text {Dom }}$, the median metallicity of the accreted stellar component is about 0.15 dex lower than the metallicity of the dominant progenitor. Although there is an increase in scatter in the contribution of the dominant progenitor to the metallicity of the accreted stellar halo at lower frac Dom $_{\text {, the }}$ scatter is $<0.1$ dex, considerably smaller than the $\sim 0.2 \mathrm{dex}$ scatter of the accreted metallicity-mass relation. This is consistent with the fact that at lower frac ${ }_{\text {Dom, a number of }}$ progenitors contribute to the build up the accreted stellar component, leading to an increase in scatter in the accreted metallicity-stellar mass relationship. From Figures 7 and 8, we conclude that while there are a range of possible accretion paths to reach a given accreted stellar mass, the metallicity of the accreted stellar component is largely set by the dominant progenitor.

The scatter in accreted metallicity can inform us about the mass of the dominant progenitor. If the accreted stellar component was built from a single massive progenitor, the metallicity of the accreted stellar component would be given by the Illustris galaxy metallicity-stellar mass relationship. This motivates us to define the quantity $[\mathrm{Fe} / \mathrm{H}]_{\text {diff }}$, i.e., the difference between the median accreted metallicity and the metallicity of the accreted stellar component if it is built up from a single massive progenitor $\left([\mathrm{Fe} / \mathrm{H}]_{\mathrm{diff}}=\right.$ $\left.[\mathrm{Fe} / \mathrm{H}]_{\text {acc }}-[\mathrm{Fe} / \mathrm{H}]_{\text {acc predict }}\right)$, where $[\mathrm{Fe} / \mathrm{H}]_{\text {acc predict }}$ is the stellar metallicity assuming the accreted stellar component was built from a single massive progenitor. We estimate $[\mathrm{Fe} / \mathrm{H}]_{\text {acc predict }}$ using the Illustris galaxy metallicity-stellar mass relationship, assuming that $\mathrm{M}_{*} \sim \mathrm{M}_{\mathrm{acc}}$. Given the 


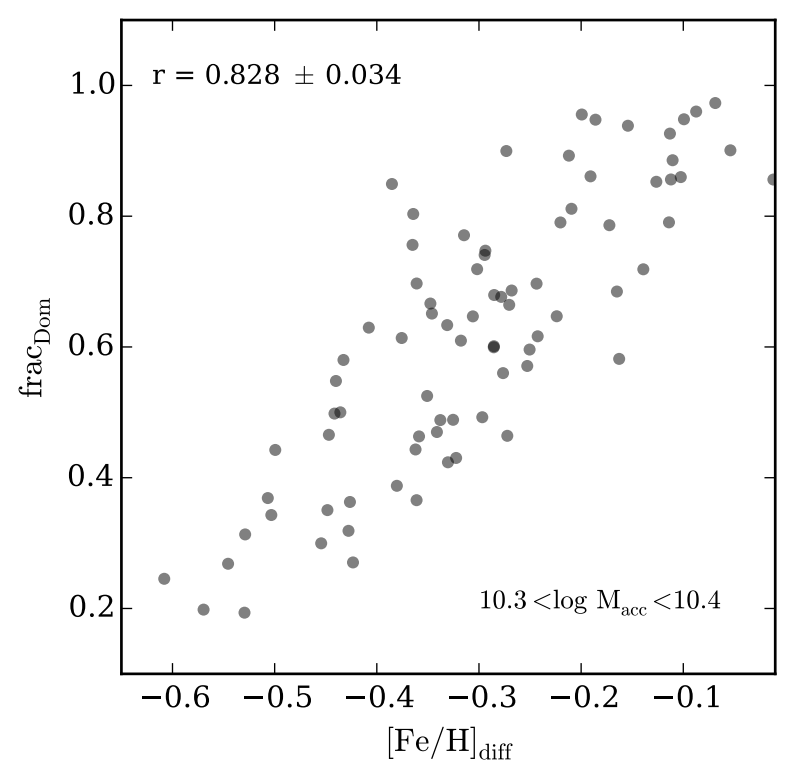

Figure 9. The fraction of accreted stellar material contributed by the dominant progenitor frac ${ }_{\text {Dom }}$ as a function of $[\mathrm{Fe} / \mathrm{H}]_{\text {diff }}=$ $[\mathrm{Fe} / \mathrm{H}]_{\mathrm{acc}}-[\mathrm{Fe} / \mathrm{H}]_{\text {acc predict }}$ at a fixed accreted stellar mass $\left(10.3<\log \mathrm{M}_{\mathrm{acc}}<10.4\right)$. The difference in accreted stellar metallicity $\left([\mathrm{Fe} / \mathrm{H}]_{\text {diff }}\right)$ encodes information about frac ${ }_{\text {Dom }}$.

ansatz that the metallicity of an accreted galaxy only depends upon its stellar mass, this quantity $[\mathrm{Fe} / \mathrm{H}]_{\text {diff }}$ allows us to quantify frac ${ }_{\text {Dom }}$ in terms of metallicity. In Figure 9, we plot frac Dom as a function of $[\mathrm{Fe} / \mathrm{H}]_{\text {diff }}$ at a fixed accreted stellar mass $\left(10.3<\log \mathrm{M}_{\mathrm{acc}}<10.4\right)$. There is a strong correlation between frac $\mathrm{C}_{\mathrm{Dom}}$ and $[\mathrm{Fe} / \mathrm{H}]_{\mathrm{diff}}$, allowing us to predict the mass of the dominant progenitor at a given accreted stellar mass.

\section{SCATTER IN THE ACCRETED METALLICITY-STELLAR MASS RELATIONSHIP AT FIXED VIRIAL SIZE}

At a fixed accreted stellar mass, galaxies span a range in virial masses and hence radii (see Figure 5). In order to explore the scatter in the accreted-metallicity stellar mass relationship, it is imperative to control for galaxies of similar sizes. We do this in this section by comparing galaxies in a narrow DM halo mass range. This allows connections to be made between our work and that of e.g., Bullock \& Johnston (2005), Deason et al. (2016), Amorisco (2017a) or Monachesi et al. (in preparation). As seen in Figure 5, galaxies belonging to a narrow DM halo mass range — such as MW-like mass galaxies - occupy a narrower and steeper locus on the accreted metallicity-stellar mass plane (illustrated by the fits to narrow DM halo mass ranges, shown as thin solid lines, in Fig. 5). We note that similar steeper relationships between accreted metallicity and stellar mass of the halo are found if one considers narrow ranges in stellar mass or velocity dispersion.

For the purposes of this study, we examine MW-like

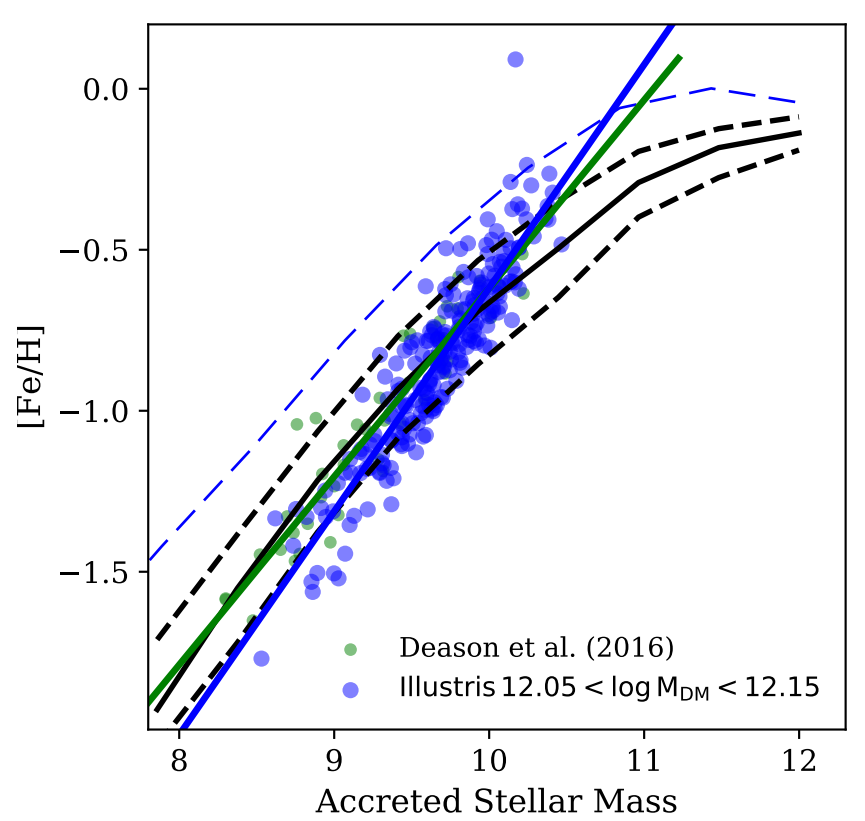

Figure 10. Comparison of the 45 haloes from the Deason et al. (2016) models and Illustris galaxies in the narrow DM halo mass range $\left(12.05 \leqslant \log \mathrm{M}_{\mathrm{DM}} \leqslant 12.15\right)$. The solid blue and green lines indicate linear fits to the Illustris and Deason et al. models respectively. The red solid and dashed lines indicate the median and the $16 / 84$ th percentile of the parent distribution of central Illustris galaxies as shown in Figure 5. The blue dashed line indicates the median metallicity-stellar mass relationship of galaxies in the Illustris simulations.

mass galaxies in DM halo mass range $\left(12.05 \leqslant \log \mathrm{M}_{\mathrm{DM}} \leqslant\right.$ 12.15). Before we begin comparing the physical properties of MW-like mass galaxies, we examine the accreted metallicitystellar mass relationship for these galaxies. In Figure 10, we plot the accreted metallicity-stellar mass relationship of the Illustris galaxies for MW-like mass galaxies in blue. We note that the slope of the accreted metallicity-stellar mass relationship for MW-mass like galaxies is much steeper than the Illustris metallicity-stellar mass relationship for all galaxies (blue dashed line).

We also compare these galaxies with the metallicity of total accreted stellar component of the 45 model stellar haloes from Deason et al. (2016) in green. We find that while the results agree in overall normalisation, they have a small difference in slopes with the Illustris galaxies producing a steeper accreted metallicity-stellar mass relationship than Deason et al.. This is a direct consequence of the difference in the input stellar mass-halo mass and metallicitystellar mass relationships between the Illustris simulations and Deason et al. (2016). In the latter, the stellar mass is assigned to DM haloes with the relationship published in Garrison-Kimmel et al. (2014), while the metallicity assigned to the stellar particles follows Kirby et al. (2013) with explicit time-evolution derived from the simulations of $\mathrm{Ma}$ et al. (2016). On the other hand, the Illustris simulations has a much steeper galaxy metallicity-stellar mass relationship with hardly any time evolution.

In a narrow DM halo mass range, the accreted stel- 
lar components of these galaxies show a rich diversity in accreted stellar mass. The top panel of Figure 11 shows the distribution of accreted stellar masses for MW-like mass galaxies. We note that the measured accreted stellar mass of the MW puts it at the tail end of the distribution of accreted stellar mass, highlighting its unusual accretion history.

The bottom panel of Figure 11 quantifies the relationship between the stellar mass of the dominant progenitor to the accreted stellar mass for MW-like mass galaxies, colourcoded with the time of accretion of the dominant progenitor. The dashed line shows frac $_{\text {Dom }}=1$; galaxies below this line have frac ${ }_{\text {Dom }}<1$. Galaxies with smaller accreted stellar mass have significantly smaller dominant progenitors (smaller frac $_{\text {Dom }}$ ), consistent with the idea that their accreted stellar component was built up through a number of accretion events. Given galaxies of a similar DM halo mass, there exists a scattered correlation between frac ${ }_{\text {Dom }}$ and the accreted stellar mass with a significant scatter, consistent with the results of Deason et al. (2016) and Amorisco (2017b).

For galaxies of similar DM halo mass, the dominant progenitors of galaxies with lower frac Dom $_{\text {are accreted earlier }}$ in time, while larger dominant progenitors are accreted later in time (as seen in Figure 11). While there exists a correlation between the mass of the dominant progenitor and the time of its accretion, there is a considerable scatter in the relationship.

Additional information is needed to break the degener-

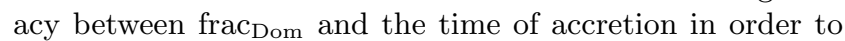
infer the stellar mass and the time of accretion of the dominant progenitor. This information may be contained in the other physical properties of the accreted stellar component (including its morphology), to which we turn to in Section 5.1 .

Even with the additional constraint of the DM halo mass of the galaxy, it is instructive to ask which quantity, the accreted stellar mass or the accreted stellar metallic-

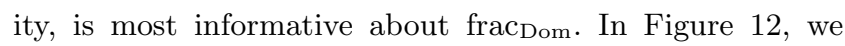

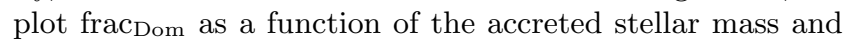
$[\mathrm{Fe} / \mathrm{H}]_{\text {diff. }}$. From the Pearson correlation coefficients, we confirm that the accreted stellar metallicity (or $[\mathrm{Fe} / \mathrm{H}]_{\text {diff }}$ ) is more informative about frac Dom $_{\text {than }}$ the accreted stellar mass for MW-like mass galaxies. This is consistent with the results found in Section 4 at a fixed accreted stellar mass.

To set further constraints on frac $_{\text {Dom }}$, it is best to compare MW-like mass galaxies at a fixed accreted stellar mass. We do this in the right panel of Figure 12, where we plot frac $_{\text {Dom }}$ as a function of the accreted stellar mass colourcoded by $[\mathrm{Fe} / \mathrm{H}]_{\text {diff }}$. We see that at a given accreted stellar mass, frac Dom correlates with $[\mathrm{Fe} / \mathrm{H}]_{\text {diff }}$ allowing us to distinguish galaxies according to the mass of their dominant progenitor. The best predictor of frac $\mathrm{Dom}_{\mathrm{D}}$ is the accreted stellar metallicity at a fixed accreted stellar mass.

It is important to note that while $[\mathrm{Fe} / \mathrm{H}]_{\text {diff }}$ correlates best with frac ${ }_{\text {Dom }}$, the accreted stellar metallicity $\left([\mathrm{Fe} / \mathrm{H}]_{\mathrm{Acc}}\right)$ is the best predictor of $\mathrm{M}_{\mathrm{Dom}}$, through the galaxy metallicity-stellar mass relationship (See Figure 13). The scatter in the relationship between $[\mathrm{Fe} / \mathrm{H}]_{\mathrm{Acc}}$ and $\mathrm{M}_{\mathrm{Dom}}$ is the same as the scatter in the Illustris galaxy metallicitystellar mass relationship.

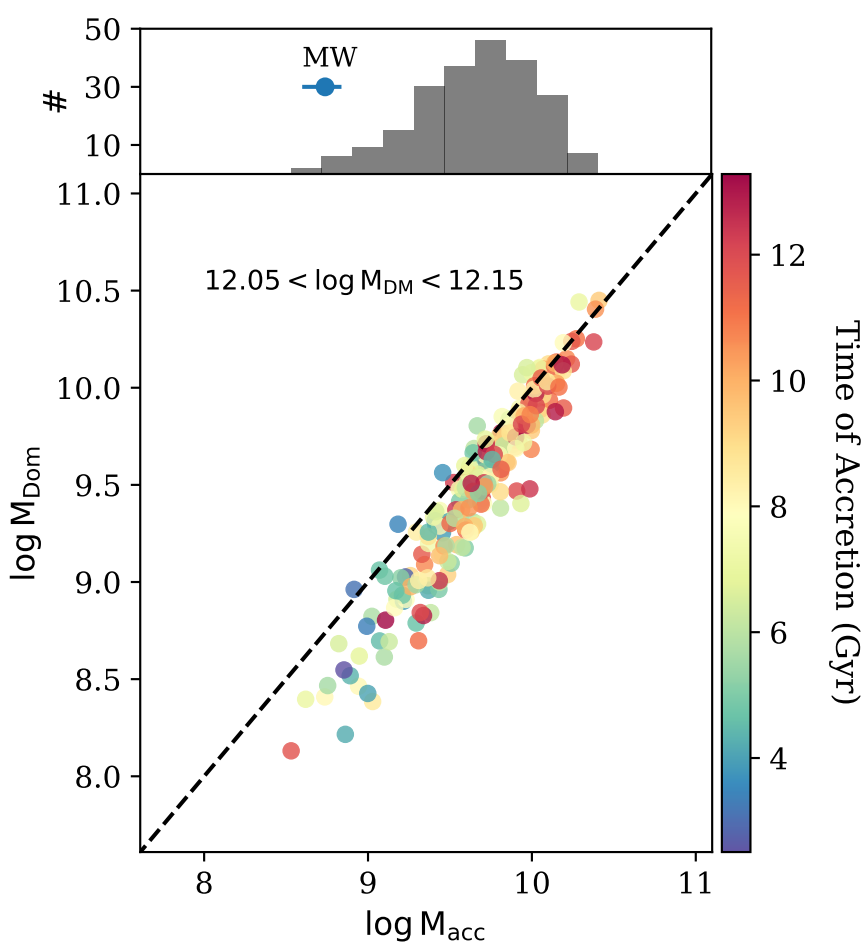

Figure 11. Top panel: The distribution of accreted stellar masses for galaxies with DM halo masses, $12.05 \leqslant \log \mathrm{M}_{\mathrm{DM}} \leqslant$ 12.15. The accreted stellar mass of the MW is indicated (BlandHawthorn \& Gerhard 2016, following Bell et al. 2008) and is at the lower end of the distribution of MW-mass galaxies, hinting to a quiet accretion history. Bottom panel: The stellar mass of the dominant progenitor as a function of the accreted stellar mass, colour coded by the time of accretion of the progenitor, where red signifies early accretions at high redshift and blue signifies a dominant accretion close to the present day. The dashed line shows frac $_{\text {Dom }}=1$; galaxies below this line have frac ${ }_{\text {Dom }}<1$. In general, larger progenitors are accreted much later in time.

\subsection{Diversity of physical properties of the accreted stellar component of MW-like mass galaxies}

Due to dynamical friction and tidal stripping, the accreted stellar component of MW-like mass galaxies display a large diversity in their physical morphology. The influence of dynamical friction on the deposition of tidally shredded material from incoming satellites in the accreted stellar component of a galaxy have been explored in depth by Amorisco (2017a) using idealised N-Body simulations and RodriguezGomez et al. (2016) for the Illustris galaxies. In general, the deposition of accreted stellar material within the hosts depends upon the mass of the progenitor, its compactness and the time of its accretion. More massive progenitors deposit their stellar material at smaller radii deep within the host, while less massive progenitors deposit their stellar material at the outskirts of the galaxies. Satellites accreted at a higher redshift deposit their stellar material at smaller radii within the host, since the hosts were physically smaller at that time. More concentrated progenitors deposit their material at smaller radii. In particular, Amorisco (2017a) showed that due to the mass-concentration scatter in DM 

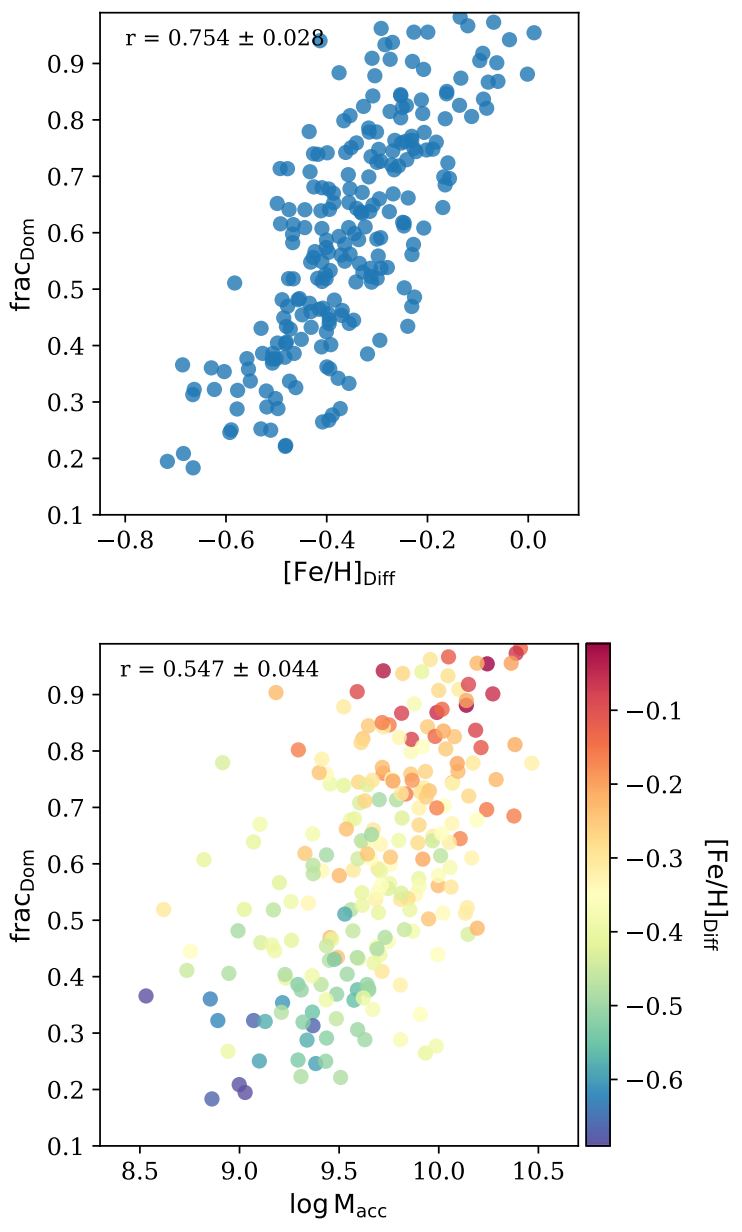

Figure 12. Top Panel: frac ${ }_{D o m}$ as a function of $[\mathrm{Fe} / \mathrm{H}]_{\text {diff }}$ for MW-like mass galaxies. Bottom Panel: frac $\mathrm{Dom}_{\mathrm{D}}$ as a function of the accreted stellar mass colour-coded by $[\mathrm{Fe} / \mathrm{H}]_{\text {diff }}$ for $\mathrm{MW}$-like mass galaxies. We also calculate the Pearson Correlation coefficient $(\mathrm{r})$ for the two relationships. The best predictor of frac ${ }_{\mathrm{Dom}}$ of MW-like mass galaxies is the accreted stellar metallicity at a fixed accreted stellar mass.

haloes, satellites that are $1 \sigma$ more concentrated than average can deposit their stars at radii that are closer in by a factor of $\sim 2.5$ in mass (and average concentration). It would be difficult to disentangle the mass of the progenitor or the time of its accretion purely from spatial information of the accreted stellar component.

In Figure 14, we demonstrate the diversity in the physical properties of the accreted stellar component for MW-like mass galaxies found in the Illustris simulations. In particular, we show the $3 \mathrm{D}$ half-mass radius $\left(\mathrm{R}_{50 \text { acc }}\right)$, the power-law density slope $\left(\Gamma_{\min \text { acc }}\right)$ and the metallicity gradient along the minor axis of the accreted stellar component. We plot these quantities as a function of the accreted stellar metallicity of the galaxy.

First of all, we find a rich diversity in the physical morphology of the accreted stellar component. Galaxies with a larger accreted stellar metallicity tend to have more compact accreted stellar component (smaller $R_{50 \text { acc }}$ ) than galaxies with a lower accreted stellar metallicity. Similarly, both the power-law density slope and the slope of the accreted metal-

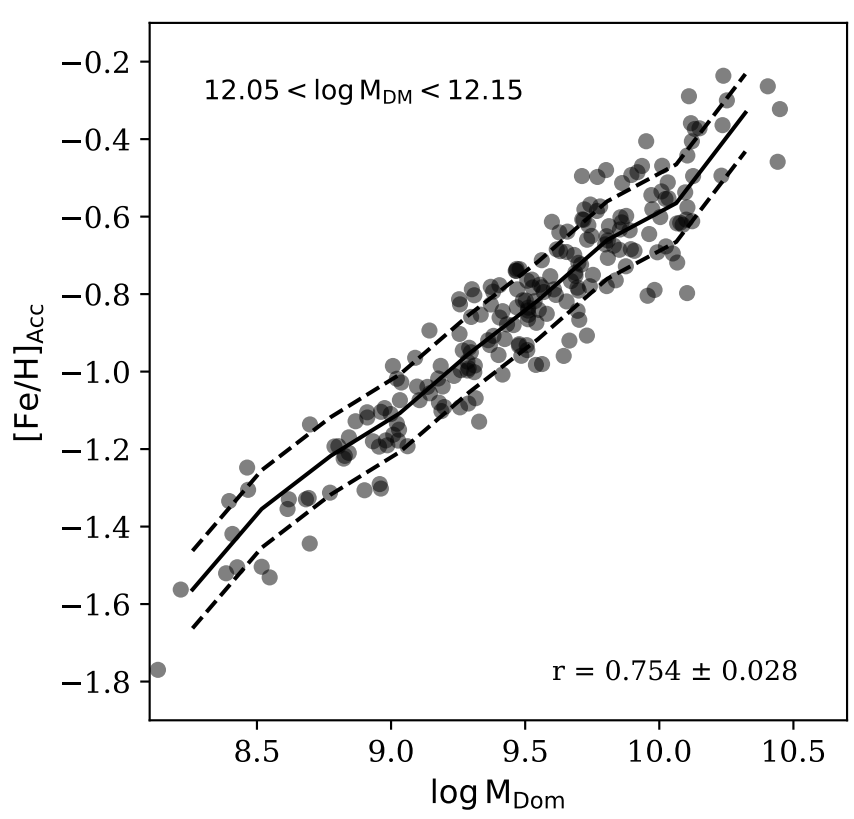

Figure 13. The relationship between the accreted stellar metallicity and the mass of the dominant progenitor for MW-like mass galaxies. The relationship follows the Illustris galaxy metallicitystellar mass relationship seen in Figure 1. The dashed lines indicate the 1- $\sigma$ scatter in the relationship. We calculate the Pearson correlation coefficient ( $r$ ) of the relationship. The accreted stellar metallicity is a strong predictor of the stellar mass of the dominant progenitor.

licity gradient becomes steeper with increasing accreted stellar metallicity. The decreasing trend in all these relationships with increasing accreted stellar metallicity reflects the correlation of increasing accreted stellar metallicity with the mass of the dominant progenitor $\left(\mathrm{M}_{\text {Dom }}\right.$; see Figure 13$)$. Galaxies with larger dominant progenitors $\left(\mathrm{M}_{\text {Dom }}\right)$ tend to have more compact accreted stellar components.

Secondly, there is also a significant scatter in these quantities at fixed accreted stellar metallicity. At lower accreted stellar metallicity, there is a substantial spread in the physical size of the accreted stellar component. The scatter in the relationships decreases with increasing accreted stellar metallicity. This is because the physical properties of the accreted stellar component are being dominated by a single large progenitor, due to increasing frac Dom $_{\text {with increasing }}$ accreted stellar metallicity.

Finally, the scatter in these relationships at a fixed accreted stellar metallicity correlates with the time of accretion of the dominant progenitor satellite (see colour-coding in Figure 14). In general, satellites accreted earlier in time deposit their stellar material at smaller radii within the host, because their hosts are much smaller. Concentrated progenitors also deposit their disrupted material at smaller radii. Moreover, the concentration of DM haloes reflects the matter density of the Universe at the time of their formation (Navarro et al. 1997). Accreted DM haloes formed earlier tend to be more concentrated and sink more towards the centre of the galaxy due to dynamical friction, than accreted DM haloes formed later which tend to be less con- 

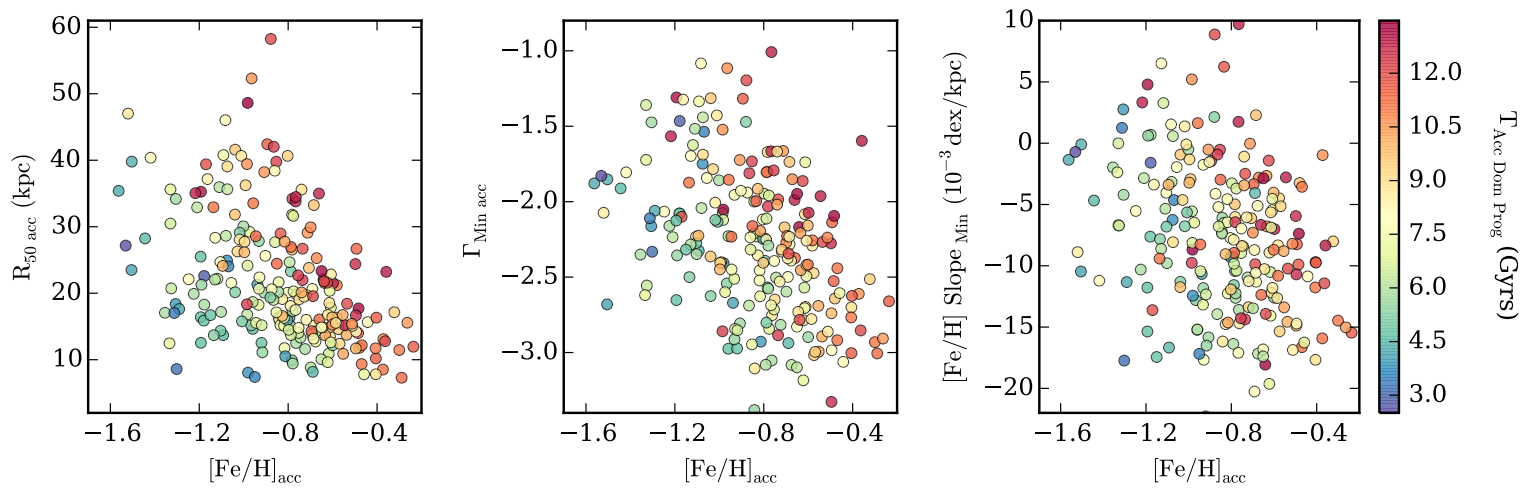

Figure 14. The diversity of the accreted stellar component of MW-like mass galaxies: The 3D half-mass radius, the power-law density slope and the metallicity gradient along the minor axis of the accreted stellar component as a function of the accreted stellar metallicity for MW-like mass galaxies. The galaxies are colour-coded by the time of accretion of the dominant progenitor, where red signifies early accretions at high redshift and blue signifies a dominant accretion close to the present day. The physical size, the surface mass density slope as well as the metallicity gradient encodes information about the time of accretion of the dominant progenitor.

centrated and deposit their disrupted accreted material at large galacto-centric distances. This induces a further correlation with time, wherein satellites which are formed earlier deposit their accreted stellar material closer to the centre of the galaxy. The scatter in the physical "spatial" properties of the accreted stellar components of galaxies is informative about the time of its build-up: the physical size, the surface mass density slope as well as the metallicity gradient of the accreted stellar component encodes information about the time of accretion of the most dominant progenitor.

We quantify this further in Figure 15 by choosing galaxies with the similar masses of the dominant progenitor. We do so by choosing galaxies using the more observationally accessible quantity $[\mathrm{Fe} / \mathrm{H}]_{\text {acc }}$ (see Section 6 ) employing the correlation between $[\mathrm{Fe} / \mathrm{H}]_{\mathrm{acc}}$ and $\mathrm{M}_{\mathrm{Dom}}$ (see Figure 13). In Figure 15 , we choose galaxies such that $-1<[\mathrm{Fe} / \mathrm{H}]_{\text {acc }}<-0.8$. We find that the $3 \mathrm{D}$ half-mass radius correlates most with the time of accretion of the dominant progenitor. We also notice that the other indicators indicating spatial information, that is, the $2 \mathrm{~d}$ power-law density slope and the metallicity gradients of the accreted component along the minor axis also encodes some information about the time of accretion. On the other hand, we find that accreted stellar mass is a poor predictor of the time of accretion consistent with Figure 11 . We also note that the presence of time evolution in the galaxy metallicity-stellar mass relationship will further affect the metallicity gradients. This effect cannot be studied in the Illustris simulation.

On the other hand, spatial information encoded in the physical properties of the accreted stellar component con-

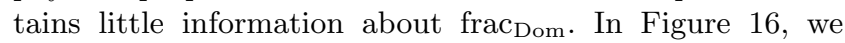
demonstrate that for a narrow accreted stellar mass range $\left(9.5<\log \mathrm{M}_{\mathrm{acc}}<9.7\right.$ for MW-like mass galaxies $)$, frac ${ }_{\mathrm{Dom}}$ correlates most with $[\mathrm{Fe} / \mathrm{H}]_{\text {diff }}$ in comparison to other indicators involving spatial information. This confirms that the spatial physical properties of the accreted stellar halo contained in the power-law density slope and metallicity gradient cannot constraint frac ${ }_{\text {Dom }}$ alone.

\section{$5.2 \quad$ Summary}

In general, we have demonstrated that it is possible to constrain the characteristics of the dominant progenitor in terms of its stellar mass as well as the time of its accretion. The accreted stellar metallicity can best constrain the mass of the dominant progenitor, while the physical spatial information of the accreted stellar component can best constrain the time of accretion of the dominant progenitor.

\section{OBSERVABLE MEASUREMENTS ALONG THE MINOR AXIS}

We have seen how the metallicity and the mass of the total accreted stellar component can inform us about the characteristics of the dominant progenitor. Observationally, however, we have no direct access to either of these two quantities. Rather, we rely on observational proxies for the same: accreted stellar metallicity estimated at a fixed distance along the minor axis and aperture measurement of the mass of the accreted stellar component. In this section, we explore how we can recover the metallicity and mass of the total accreted stellar component from the observational proxies. Although we concentrate on MW-like mass galaxies for illustrative purposes, the results of this section are generalizable to galaxies spanning a broad range in masses.

\subsection{Accreted Stellar Metallicity}

MW-like mass galaxies exhibit a range of stellar metallicity gradients along the minor axis even at large galacto-centric distances (Monachesi et al. 2016). A similar trend is found in Illustris galaxies: they show a similar range in metallicity gradients along the minor axis even in the accreted component (see Figure 14). This suggests that a measurement of the metallicity of the accreted component along the minor axis at large galacto-centric distances can be considerably lower than the total accreted stellar metallicity for galaxies

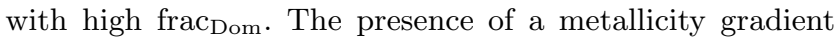
informs us that a measurement along the minor axis is not 

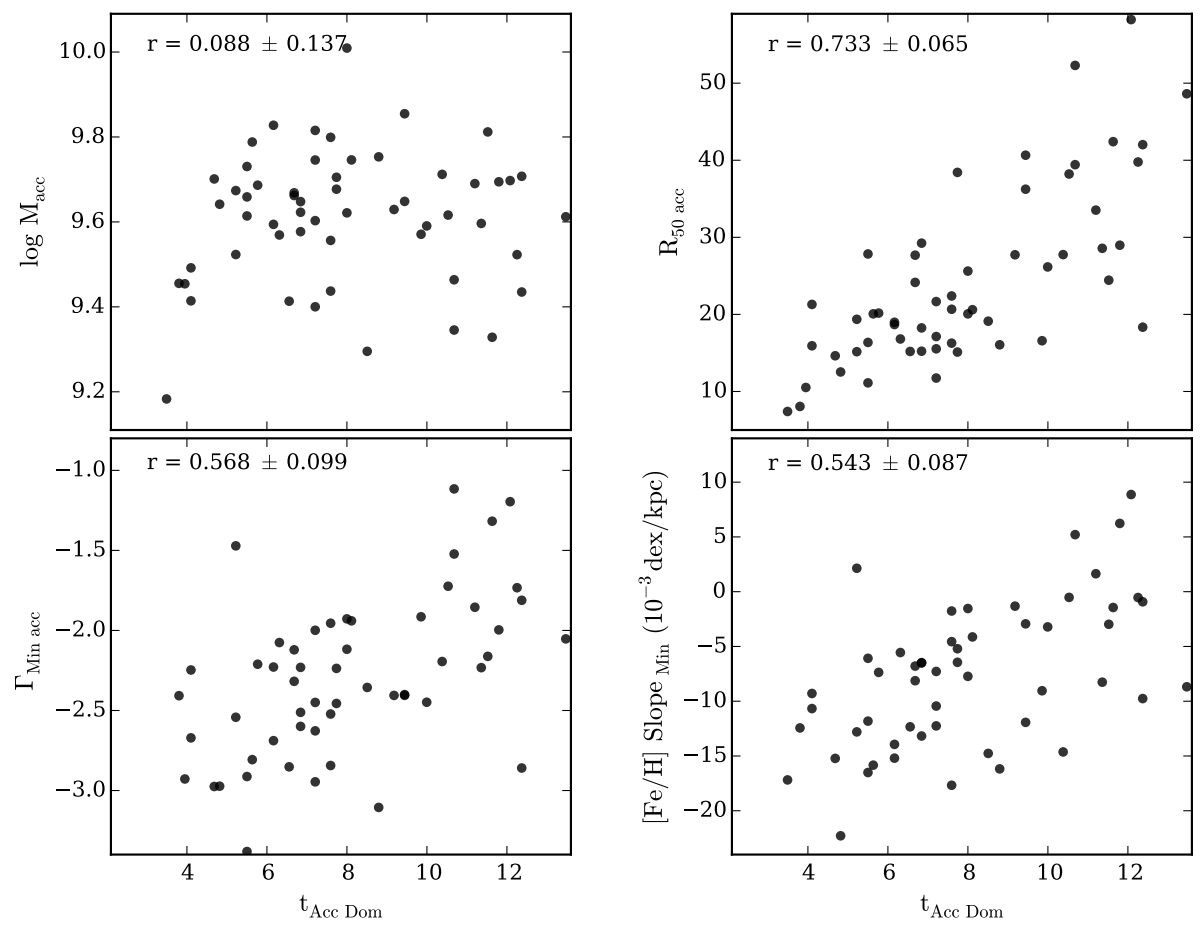

Figure 15. For MW-like mass galaxies with accreted stellar metallicites $\left(-1<[\mathrm{Fe} / \mathrm{H}]_{\mathrm{acc}}<-0.8\right)$, we plot the correlations between the time of accretion of the dominant progenitor and $\log \mathrm{M}_{\mathrm{acc}}, \mathrm{R}_{50 \text { acc }}, \Gamma_{\mathrm{acc}}$ min as well as the stellar metallicity gradient of the accreted component along the minor axis. We also calculate the Pearson Correlation coefficient (r) for the relationships. The physical size, the surface mass density slope as well as the metallicity gradient of the accreted stellar component encodes information about the time of accretion of the most dominant progenitor, while the mass of the accreted stellar component contains no information about the time of accretion.

representative of the metallicity of the total accreted stellar component, as well as about the degree of that misrepresentation. The information encoded in the metallicity gradient could allow us to reconstruct the metallicity of the total accreted stellar component from the metallicity measurement along the minor axis.

In order to directly compare our results with the estimated metallicities from the GHOSTS-like observations, we consider the median accreted stellar metallicity along a projected wedge of 30 degrees along the minor axis between a galacto-centric distance of 25 and $45 \mathrm{kpc}$.

Before we examine MW-like mass galaxies, we first plot the median metallicity of the accreted stellar particles along a projected wedge along the minor axes between 25 and $45 \mathrm{kpc}$, as a function of accreted stellar mass for all central Illustris galaxies in Figure 17. This relationship is similar to the total accreted metallicity-stellar mass relationship (compare to Figure 5): While both relationships have similar slope, the relationship along the minor $\mathrm{x}$-axis is $\sim 0.2 \mathrm{dex}$ lower. At lower accreted stellar mass $\left(<\log \mathrm{M}_{\text {acc }}\right)$, the relationship along the minor axis approaches the total accreted metallicity-stellar mass relationship. There is a considerable spread in the relationship along the minor axis, comparable to that found along the total accreted metallicity-stellar mass relationship in Figure 5. The difference in the two re- lationships can be accounted for in terms of metallicity gradients along the minor axis.

We now proceed to examine if we can reconstruct the total accreted stellar metallicity from observations along the minor axis using the metallicity gradients for MWlike mass galaxies. As a first order, we consider a simple linear extrapolation scheme such that $[\mathrm{Fe} / \mathrm{H}]_{\text {reconst }}=$ $[\mathrm{Fe} / \mathrm{H}]_{\text {minor }}+$ gradient $\times 35 \mathrm{kpc}$. In Figure 18 , we explore the ability of this simple linear extrapolation scheme to recover the metallicity of the total accreted stellar component. While there is a scatter of the reconstructed accreted metallicity around the total accreted metallicity, this scatter is around 0.1 dex. This lends support to the idea that we can reconstruct the metallicity of the total accreted stellar component within an accuracy of 0.1 dex from the information encoded in the metallicity gradients. More sophisticated extrapolation schemes using the information encoded in the density profiles could do better.

\subsection{Accreted Stellar Mass}

Because much of the accreted stellar material of a galaxy is not directly observable, measuring its total accreted stellar mass involves model-dependent assumptions. Using accretion-only models of Bullock \& Johnston (2005), Harm- 

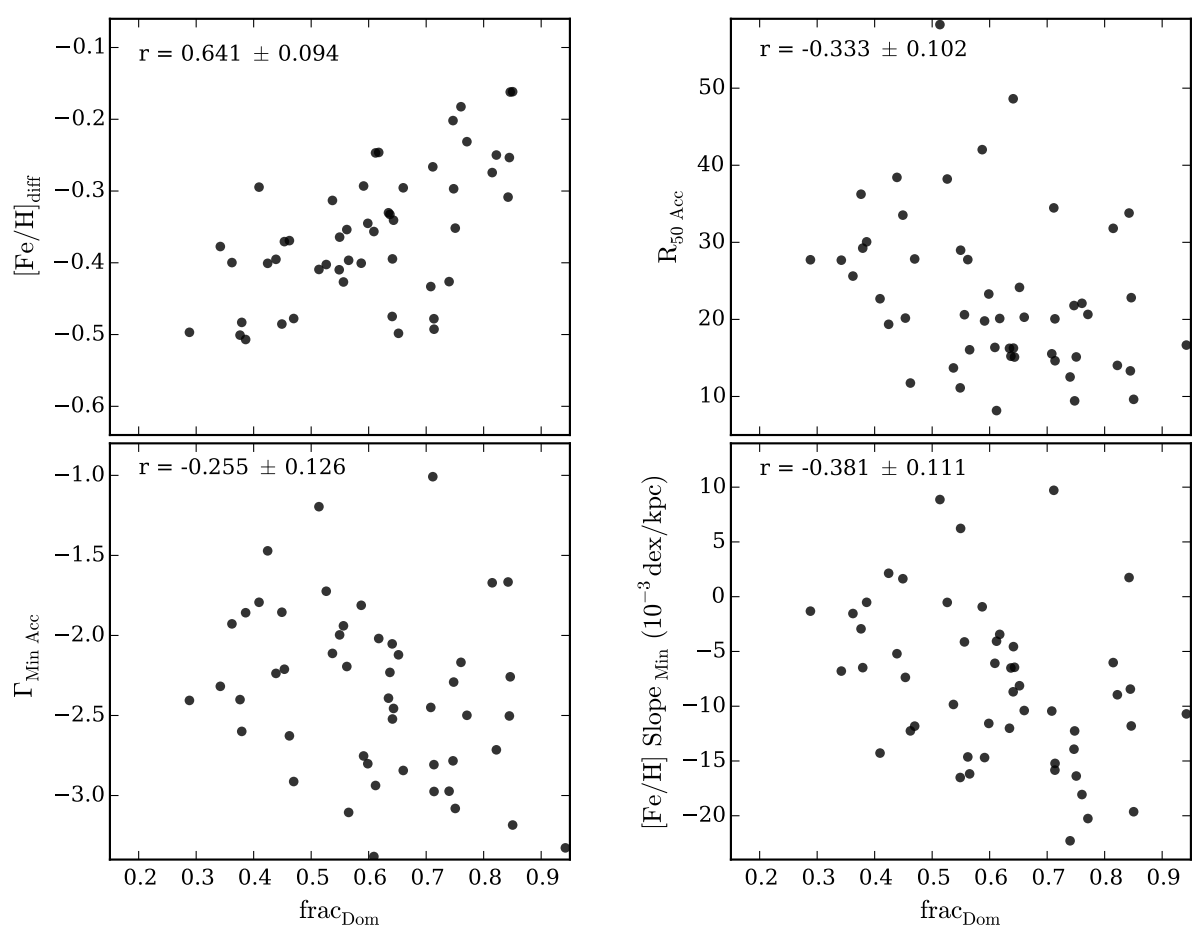

Figure 16. For MW-like mass galaxies with an accreted stellar mass in the range $9.5<\log \mathrm{M}_{\text {acc }}<9.7$, we plot the correlations between frac Dom and $[\mathrm{Fe} / \mathrm{H}]_{\text {diff }}, \mathrm{R}_{50 \text { acc }}, \Gamma_{\mathrm{acc} \text { min }}$ as well as the metallicity gradient of the accreted component along the minor axis. We also calculate the Pearson Correlation coefficient (r) for the relationships. The metallicity of the accreted stellar component is highly

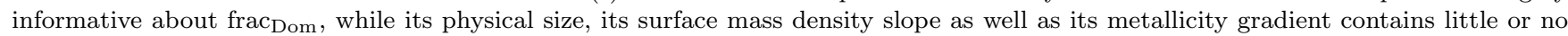
information.

sen et al. (2017) and Bell et al. (2017) extrapolated an "aperture" stellar halo mass between $10-40 \mathrm{kpc}$ along the minor axis to a total accreted stellar mass. We evaluate these extrapolations for Illustris MW-like mass galaxies. To do this, we consider a circular aperture measurement (between 15 and $50 \mathrm{kpc}$ along the minor axis, corresponding approximately to an elliptical aperture of semi-minor axis $10-40 \mathrm{kpc}$ ) of the stellar mass of the accreted halo. In Figure 19, we plot the difference between the total accreted stellar mass and the aperture measurement of the same as a function of the total accreted stellar mass. We find that the total accreted stellar component mass is $\sim 3 \times$ the "aperture" accreted mass with a scatter of $30 \%$ and a $<15 \%$ variation with accreted stellar mass. Consequently, the total accreted stellar mass can be reconstructed to within an accuracy of 0.15 dex. This validates the claims of Harmsen et al. (2017) and Bell et al. (2017), and allows us to connect the observable part of the accreted stellar component at large galacto-centric distances with the total accreted stellar material, the bulk of which is present at small radii due to dynamical friction (Cooper et al. 2013; Amorisco 2017a).

Moreover, we find that the scatter in the difference between the total and the "aperture" measurements at a fixed accreted stellar mass correlates best with the 3D half-mass radius of the accreted stellar component $\left(R_{50}\right.$ Acc $)$. Galaxies with a more extended stellar halo have a smaller aperture accreted stellar mass compared to galaxies with a less extended stellar halo. Similar correlations are also seen with the $2 \mathrm{~d}$ power-law density slope of the accreted stellar component along the minor axis $\left(\Gamma_{\text {Min acc }}\right)$ as well as its normalization $\Sigma_{0 \text { Acc }}$ (see Figure 19). As demonstrated in Section 5.1, $\mathrm{R}_{50 \text { Acc }}$ at a fixed accreted stellar mass correlates best with the time of accretion of the dominant merger.

Thus, assuming that the stellar halo structures in Illustris are reasonably accurate, we suggest that future analyses using information encoded in the minor axis density profile of the accreted stellar component may permit more robust extrapolations from an "aperture" measurement to the total accreted stellar mass of the galaxy.

\section{DISCUSSION}

In this work, we use the Illustris simulations to build intuition of how the accreted metallicity-stellar mass relationship comes about in central galaxies and how it can inform us about the properties of the dominant progenitor. A powerful advantage of Illustris over previous works (e.g., Renda et al. 2005; Font et al. 2006; Deason et al. 2016) is the large dynamic range in DM halo mass, and its use of a large cosmological volume. This allows us to explore galaxies over a broad range in accretion histories. We find the accreted 


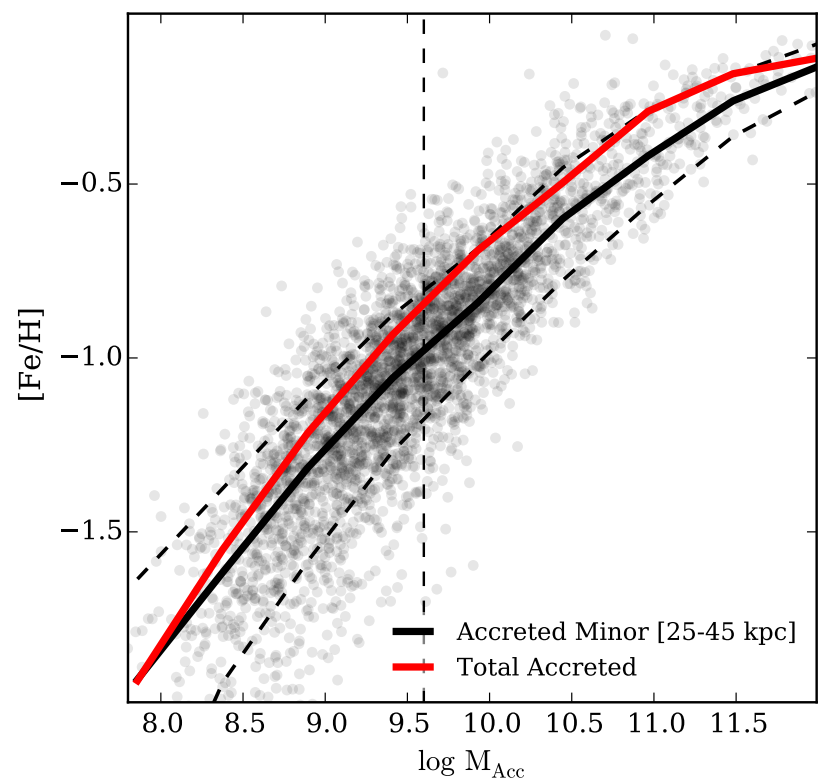

Figure 17. The median metallicity of the accreted component (estimated in wedges with 30 degrees along the minor axes between a galactocentric distance of 25 and $45 \mathrm{kpc}$ ) as a function of the accreted stellar mass of the Illustris galaxies. The black solid and dashed lines indicate the median and the 16/84th percentile of the distribution. The red solid line indicates the median of the metallicity of the total accreted stellar component. The metallicity estimated along the minor axis will be lower than the metallicity of the total accreted stellar component.

metallicity-stellar mass relationship extends over several orders of magnitude in accreted stellar mass. The large dynamic range of this relationship is truly remarkable. Due to the galaxy metallicity-stellar mass relationship, the dominant progenitor drives the metallicity of the accreted stellar halo (Deason et al. 2016). This relationship enables us to probe the complex multi-dimensional space of an accretion history of the galaxy in terms of its dominant progenitor.

We use Illustris to discuss possible observational metrics of the properties of the dominant progenitor of a given stellar halo. The metallicity of a stellar halo is driven by its dominant progenitor, and together with the total accreted mass gives an estimate of the fraction of the total stellar halo contributed by the most dominant progenitor. Metallicity and density gradients allow one to constrain the time of the dominant accretion; galaxies with earlier dominant accretions have more compact halos with steeper gradients, when compared to the ensemble of stellar halos with a given accreted mass. We use Illustris to argue that minor axis observations of resolved stars in stellar halos contain enough information to recover total accreted mass to better than 0.15 dex accuracy, and median accreted metallicity to within 0.1 dex, opening the door to applying these metrics to observational datasets.

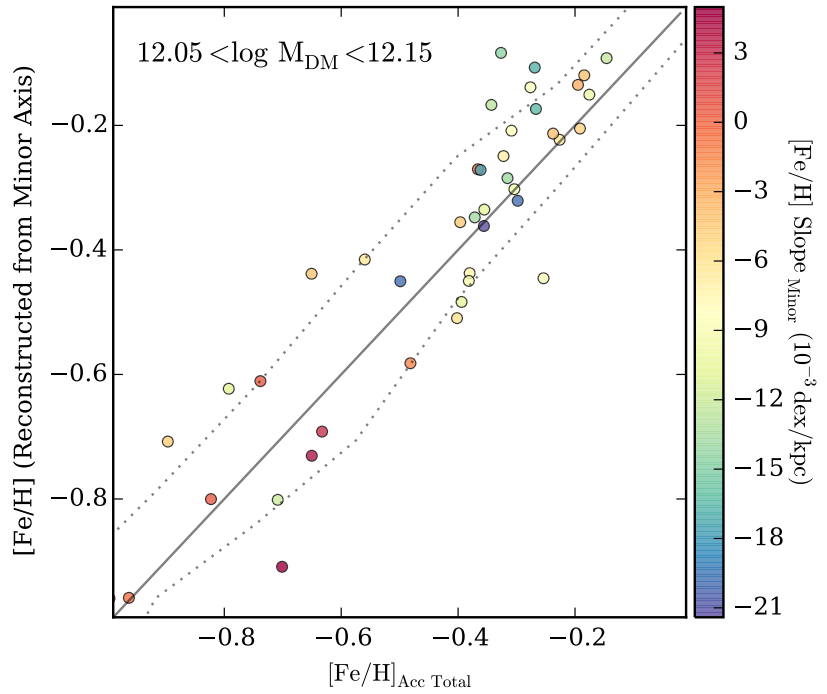

Figure 18. The reconstructed metallicity of the total accreted stellar component as a function of the true median accreted stellar metallicity colour-coded by the metallicity gradient for MW-like mass galaxies. The accreted metallicities are reconstructed using a simple linear extrapolation using the estimated accreted stellar metallicity along the minor axis at $35 \mathrm{kpc}$ as well as the information encoded in the metallicity gradients. The dashed lines shows the scatter in the relationship. The metallicity of the total accreted stellar component can be reconstructed from information about the metallicity along the minor axis.

\subsection{Limitations of this study}

The strengths of Illustris for this work are its combination of volume, resolution, dynamic range in halo masses, a reasonable satellite progenitor population and tagging of star particles as accreted or in situ. Yet, all simulations have limitations, and Illustris is no exception. Illustris has four potentially important limitations that impact our results. First, the stellar mass function (SMF) of the Illustris simulations shows an excess of low stellar mass galaxies up to redshift $z \sim 2$. Secondly, the stellar mass-halo mass relationship of the Illustris galaxies match the observational constraints imperfectly. Thirdly, the Illustris simulation has a steeperthan-observed galaxy metallicity-stellar mass relationship, exhibiting little time evolution. Finally, the Illustris galaxies fail to reproduce the observable mass-size relationship of galaxies, galaxies being fairly large for a given stellar mass (Snyder et al. 2015).

Despite these limitations, the main results of this paper are fairly robust, since we focus only on the accreted stellar component. As can be seen by comparison with e.g., Deason et al. (2016) or Amorisco (2017a), the main requirements for this analysis are a reliable dark matter framework, a reasonable stellar mass for halos at a given dark matter mass and a reasonable metallicity. Illustris satisfies these requirements easily. For MW-like mass galaxies, the bulk of the accreted stellar material is contributed by large progenitors (see also Deason et al. 2016), with DM halo masses 

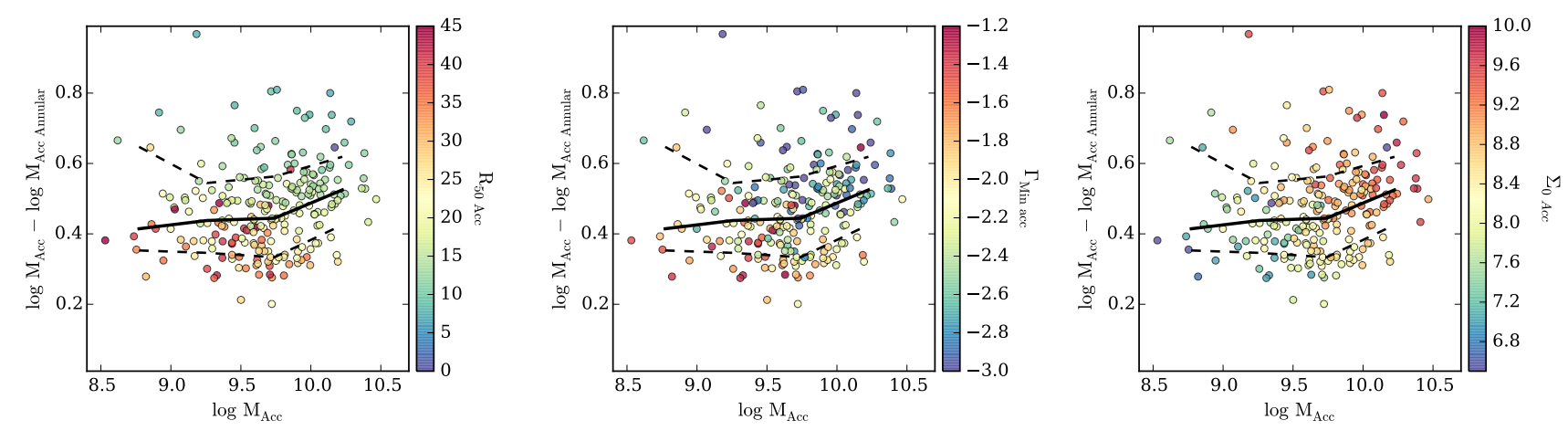

Figure 19. The difference between the total accreted stellar mass and the "aperture" measurement of the accreted stellar mass (between 10 and $50 \mathrm{kpc}$ ) as a function of the total accreted stellar mass for MW-like mass galaxies. The points are colour-coded according to 3D half-mass radius $R_{50}$ Acc $\left(\right.$ left), the $2 \mathrm{~d}$ power-law density slope of the accreted stellar component along the minor axis $\Gamma_{\text {Min acc }}$ (center), and the normalisation of the power-law fit of the density profile $\Sigma_{0 \text { acc }}$ (right). The total accreted stellar mass can be reconstructed from aperture mass measurements of the stellar halo to within an accuracy of 0.15 dex.

$\sim 4 \times 10^{11} M_{\odot}$ (Purcell et al. 2007). At this mass, Illustris reproduces the stellar-to-halo mass ratio reasonably well (Vogelsberger et al. 2014b). Smaller subhaloes deviate somewhat from the stellar mass-halo mass relation, but do not contribute sufficiently to the final stellar halos to adversely bias the accreted stellar masses of central galaxies (see Fig. 1 of Purcell et al. 2007). The Illustris simulation has a strong metallicity-mass relation. Once we adjust the normalization of the metallicity-mass relation to take into account the $[\mathrm{M} / \mathrm{H}]$ to $[\mathrm{Fe} / \mathrm{H}]$ conversion and any deficiencies in the Illustris chemical evolution framework (Fig. 1), it reproduces the metallicity of galaxies to within $\sim 0.1 \mathrm{dex}$, and the resultant stellar halos are an acceptable match to observations (Fig. 3). Moreover, the spatial distribution of the accreted stellar material is governed solely by gravity and dynamics. These suggest that the qualitative (and to a certain extent, the quantitative) results of our analysis are robust. In order to develop further our ability to quantitatively mine stellar halos for the properties of the dominant progenitor, it will be important to map out the empirical ranges of metallicity, metallicity gradients, masses and density gradients as a function of virial mass for a sizeable sample of galaxies. Relative trends will be more robust than absolute numbers, and these can be revealed only in a diverse sample which exercises the dynamic range of accreted halos. These insights may require some adjustments in the analysis of observations. For example, if the galaxy metallicity-stellar mass relationship evolves with time, $[\mathrm{Fe} / \mathrm{H}]_{\text {diff }}$ will be a function of both the mass of the dominant progenitor and the time of its accretion. In such a case, a joint analysis of the $[\mathrm{Fe} / \mathrm{H}]_{\text {diff }}$ with the spatial information of the accreted stellar component would be necessary to put constraints of the mass of the dominant progenitor and the time of its accretion.

When comparing with observations, the presence of an in situ halo (Zolotov et al. 2009; Cooper et al. 2015) could complicate the measurement of the accreted stellar metallicity. This motivates our reliance on comparisons with minor axis measurements of disk-dominated galaxies from GHOSTS (Monachesi et al. 2016; Harmsen et al. 2017), where contributions from in situ stars are expected to be negligible (Pillepich et al. 2015; Monachesi et al. 2016).
There is a substantial debate among simulators today as to the amount of in situ stars at large radius in simulated galaxies. The fraction of in situ stars present at large galacto-centric distances is highly dependent on the nature of feedback present in the hydro-dynamical simulations. The Illustris galaxies have particularly extended stellar haloes due to the presence of a large fraction of in situ stars at large galacto-centric distances (Pillepich et al. 2014). Such prominent in situ halos would over-produce the observed 10$40 \mathrm{kpc}$ stellar masses of real galaxies (Fig. 3; see also Harmsen et al. 2017). Furthermore, the flat observed metallicity gradients found in half of the GHOSTS galaxies is inconsistent with ubiquitous extended in situ halos in MW-like mass galaxies (Monachesi et al. 2016; Harmsen et al. 2017). Finally, the presence of a prominent in situ halo would reduce the slope of accreted metallicity-stellar mass relationship for MW-like mass galaxies towards the slope of the galaxy-metallicity stellar mass relationship, in seeming conflict with the observed relationship (Harmsen et al. 2017, see also Fig. 3).

Finally, because this work was motivated by the template of GHOSTS-like observations with HST, the main results of this study have restricted applicability to nearby edge-on galaxies. Realistically, the number of inclined nearby edge-on galaxies we can observe with HST are limited. New space missions including the Wide Field Infrared Survey Telescope (WFIRST) as well as the James Webb Space Telescope (JWST) will help to increase the sample size beyond the current limitations of $10 \mathrm{Mpc}$. Given the limited number of edge-on systems within this volume, it is crucial we understand how to extend these techniques to deal with face-on galaxies. Improving our modelling schemes in dealing with an in situ stellar component will help us disentangle the in situ and accreted stellar components of nearby galaxies.

\subsection{Implications and comparison with other works}

While previous works showed a relationship between the mass of an accreted halo and its metallicity (Renda et al. 2005; Font et al. 2006; Deason et al. 2016), Illustris allowed 
Stellar haloes reflect merger histories

us to characterize the accreted metallicity-stellar mass relationship over a large range in DM halo mass, accretion histories and in a cosmological volume. In accord with previous works, the main driver of this relationship is the galaxy metallicity-stellar mass relationship, where the dominant progenitor drives both the metallicity and the mass of the accreted stellar component (Deason et al. 2016).

The scale and dynamic range of Illustris allow us to explore the scatter in the accreted metallicity-stellar mass relationship, finding that Illustris predicts this scatter to be both considerable and informative. This finding was hinted at by Deason et al. (2016), but is placed on a substantially firmer qualitative and quantitative footing by Illustris. The accreted metallicity gives an informative measure of $\mathrm{M}_{\mathrm{Dom}}$; this in practice can correspond to a wide range in $\mathrm{M}_{\text {acc }}$ depending on the merger history (Fig. 5). This is of possible importance for those wishing to characterize the total accreted mass using metallicity only; such inferences will be very uncertain without the addition of extra information (e.g., a halo mass estimate, following Fig. 5).

In this work, we chose to parametrize the accretion history of a galaxy in terms of the mass and time of accretion of the most dominant progenitor. In particular, we concentrate on (frac $\left.c_{D o m}\right)$, the fraction of accreted stellar material contributed by the most dominant progenitor. There are many equivalent ways of parametrizing the accretion history of the galaxy, for example, in terms of the mean merger ratio (Rodriguez-Gomez et al. 2015; Cook et al. 2016), the mass weighted average destroyed satellite mass (Deason et al. 2016) or the number of significant progenitors which contribute a given percentage of the mass of the stellar halo (Cooper et al. 2010). Our choice of concentrating on the dominant progenitor is motivated by the fact that it drives the metallicity of the total accreted stellar component.

Previous works have argued that the properties of the largest progenitor can be constrained by using the accreted stellar mass (Deason et al. 2016; Amorisco 2017b), the slope of the density profile (Pillepich et al. 2014), or metallicity gradients (Cooper et al. 2010; Tissera et al. 2013; Hirschmann et al. 2015). Our analysis of Illustris suggests that the stellar halo metallicity is a much more direct probe of the mass of the largest progenitor, and that the scatter in accreted stellar metallicity at a given accreted stellar mass directly reflects the fraction of the accreted stellar mass ( frac $\left._{\text {Dom }}\right)$ contributed by the most dominant progenitor. We argue that while the alternative metrics suggested by other authors do correlate with dominant progenitor mass, such correlations are contributed to by confounding quantities. At a given accreted stellar mass, one can have a range in frac Dom (see Figure 11). Both the density profiles and the metallicity gradients are dependent not only on the mass of the progenitor but also on the concentration of its DM halo and the time of its accretion. On the other hand, the metallicity of the total accreted stellar component depends on the mass of the progenitor alone. We note that this result is somewhat sensitive to time evolution of the galaxy stellar metallicity-stellar mass relation; if this relation evolves, a joint analysis of the stellar halo metallicity in conjunction with its gradients will be necessary to jointly constrain the mass and time of accretion of the dominant progenitor.

For galaxies where it is difficult to estimate its accreted stellar mass (e.g. early-types or face-on disk galaxies), a mea- surement of the total accreted stellar metallicity can still constrain the mass of the dominant progenitor, for galaxies in a fixed stellar mass range (see Figure 13). If there is an evolution in the galaxy metallicity-stellar mass relationship with time, upper limits can be set on the mass of the dominant progenitor.

Recently, Amorisco (2017b) showed that accreted stellar mass in addition to the number of distinct surviving massive satellites can also provide constraints on the accretion history of the galaxy. Deason et al. (2016) also explore similar ideas. This requires surveying around the galaxy for its massive satellites in addition to measuring its accreted stellar component. This may be viable for nearby galaxies using wide-field observations of diffuse light (Merritt et al. 2016) or resolved stars (Carlin et al. 2016).

Dynamical friction affects the radial mass distribution of tidally stripped material (Amorisco 2017a). Large accreted satellites deposit their material at the centre of the galaxy, while smaller progenitors are preferentially deposited at large galacto-centric distances, affecting the density profiles of accreted stars. This leads to a preferential metallicity segregation, visible in metallicity gradients in the accreted stellar component.

We find that quantities encoding spatial information of the accreted stellar halo including the power-law density profile and metallicity gradient of the accreted stellar component becomes steeper with increasing mass of the dominant progenitor (see Figure 14). This is consistent with the analysis of Amorisco (2017a). Although the spatial information (density slopes and metallicity gradients) is primarily driven by frac ${ }_{\text {Dom }}$ (or increasing accreted stellar mass), the large scatter in these relations do not allow us to predict the mass of the most dominant satellite using purely spatial information alone. Rather, we find that at a given accreted stellar mass, the scatter in the spatial information correlates best with the time of accretion of the dominant progenitor. Galaxies which accrete earlier in time tend to have a more compact and steeper accreted stellar components than those that accrete later in time, at a fixed accreted stellar mass. These results are also consistent with the particle-tagging simulations of Cooper et al. (2010) which showed that their metallicity gradients of the accreted component correlate with the mass of the dominant progenitor.

The fundamental drivers of metallicity gradients in the accreted stellar component are the intrinsic metallicity gradients in the massive accreted satellites. The massive accreted satellites in the Illustris simulations have metallicity gradients leading to gradients in the accreted stellar haloes of galaxies. Similarly, in the simulations of Cooper et al. (2010), due to the particle-tagging methodology employed there, the massive accreted satellites had metallicity gradients. On the other hand, the particle-tagging simulations of Bullock \& Johnston (2005) showed hardly any metallicity gradients in the accreted stellar component (Font et al. 2006). This can be traced back to the limitations of the tagging procedure used in these simulations: the accreted satellites did not possess any intrinsic metallicity gradients.

It would be interesting to compare the metallicity gradients of the accreted stellar component of the Illustris MW-like galaxies with the observational constraints. Since Monachesi et al. (2016) did not publish any metallicity gradients for the GHOSTS galaxies, we cannot undertake a 
quantitative comparison. However, their published colour gradients allows us a qualitative comparison. Their colour gradients span from 0 to $-0.004 \mathrm{mag} / \mathrm{kpc}$ (Gilbert et al. 2014). M31 is one of those galaxies with an extremely large metallicity gradient. This suggests that the gradients in the GHOSTS galaxies span from 0 to $-0.01 \mathrm{dex} \mathrm{kpc}^{-1}$. From the right panel of Figure 14, we find that the metallicity gradients of the accreted stellar component of MW-like Illustris galaxies spans 0.005 to $-0.015 \mathrm{dex} \mathrm{kpc}^{-1}$. This is consistent with the metallicity gradients of the GHOSTS galaxies.

\subsection{How do these results apply to systems with a substantial in situ stellar halo?}

In order to relate these results to a broader set of observations, one needs to include the modelling of in situ stellar material. Because the results presented in this work are only valid for accretion-only systems, our best bet for observing these trends are with GHOSTS-like HST observations of the minor axis of nearby edge-on galaxies. However, some of the results of this work may also be valid for other galaxies in the limit of large galacto-centric distances, which are dominated by accreted-only stars.

Early cosmological hydrodynamical simulations had a hard time reproducing realistic galaxies, and correspondingly, the in situ component. Nevertheless, much progress has been made in recent years. Font et al. (2011) showed the observable metallicity gradients in the inner part of the galaxies $(<30 \mathrm{kpc})$ are related to the relative fraction of in situ to accreted stellar material (or the accreted stellar fraction). Cooper et al. (2013) using particle-tagging simulations extended this analysis and showed for a range in stellar masses that the shape of the density profile encodes information about the fraction of accreted stellar material. Pillepich et al. (2014) obtained qualitatively similar results using the Illustris hydrodynamical simulations by parametrizing the density profile with a power-law fit. Galaxies with a higher accreted stellar fraction have a shallower slope.

However, the question of the ability of "observable" density profiles and metallicity gradients in galaxies (involving contamination of in situ stellar material) to differentiate between possible accretion histories of a galaxy (in terms of merger fraction or time of accretion) is an open one and subject to much debate, especially in the limit of large galactocentric distances. While a number of hydrodynamical simulations show that the density profiles and the metallicity gradients of the accreted stellar component encodes information of the accretion history of the galaxy (Font et al. 2011; Tissera et al. 2013; Hirschmann et al. 2015), they differ in the predictions of their in situ stellar component and its radial extent, and how much it dominates the overall profiles.

In simulations which over-predict the amount of the in situ stellar halo, analyses of their radial profiles at smaller radial scales ( $<30 \mathrm{kpc}$ ) will be biased towards washing out any signal of the accretion history of the galaxy. Indeed, Cook et al. (2016) find that the metallicity gradients and the density slopes (between $2-4 R_{1 / 2}$ ) of quiescent Illustris galaxies are only sensitive to the accreted stellar fraction.

On the other hand, analyses which extend to larger radii where the accreted stellar material is predominant find contrasting results. Hirschmann et al. (2015) find that the metallicity gradients of quiescent galaxies (between 2-6 $R_{1 / 2}$ ) are correlated with the merger-mass ratio (Hirschmann et al. 2013). It must also be noted that the simulations of Hirschmann et al. (2013) do a better job in reproducing the mass-size relationship of galaxies in contrast to the Illustris simulations (Snyder et al. 2015). Recent simulations of disk galaxies also indicate that the observable outer metallicity gradients are indeed correlated with the accretion history of the galaxy (Tissera et al. 2013).

We conclude that at large radii (between 4-6 $R_{1 / 2}$ ) where galaxies are dominated by their accreted stellar material, the observable density profiles and the metallicity gradients at these distances continue to retain information about the accretion history of a galaxy. At intermediate distances (between 2-4 $R_{1 / 2}$ ), the accretion information content of the density profiles and metallicity gradients of galaxies depends upon the amount of in situ stellar halo being produced in the simulations.

\subsection{Reflections on the Milky Way, M31 and NGC $\mathbf{5 1 2 8}$}

While future works will explore the implications of our results on the stellar halo measurements for individual nearby galaxy merger histories in considerably more depth (see, e.g., Bell et al. 2017 for a first attempt), it is interesting to consider some implications for our understanding of the Milky Way, M31 and NGC 5128. We are on firmer ground making inferences about the Milky Way and M31, as GHOSTS provides an observed sample of peer disk galaxies, allowing relative statements to be made. In particular, the Milky Way's and M31's properties are relatively extreme, lying at the low and high-mass ends of the distribution (e.g., Fig. 3). For NGC 5128, no such sample of peers exists, limiting our ability to make strong inferences about accretion history.

\section{The Milky Way}

As many measurements of the MW stellar halo's properties do not incorporate Sagittarius, or account for it only incompletely, we discuss the Milky Way neglecting Sagittarius first, then reflect on its possible importance at the end.

The MW distinguishes itself amongst nearby galaxies with similar total stellar masses by having a very small stellar halo, consistent with a low accreted stellar mass. The total accreted stellar mass of the MW has been estimated to be $\mathrm{M}_{\text {Acc }} \sim 4-7 \times 10^{8} \mathrm{M}_{\odot}$ (Bland-Hawthorn \& Gerhard 2016, following Bell et al. 2008). Such a low accreted stellar mass is consistent with the idea that our Galaxy has been largely undisturbed over the past several gigayears and has accreted very little compared to similar sized galaxies. The accretion history of the Galaxy is more an exception than the norm for MW-like mass galaxies, lying at the low tail of the distribution of accreted stellar mass (see Figure 11). We do not expect to find many galaxies with similar sized stellar haloes, but there may still be some analogues to the MW in the local volume. M101 with an extremely small stellar halo (van Dokkum et al. 2014; Merritt et al. 2016) might be a MW-equivalent in terms of its sparse accretion history.

On the other hand, the MW halo also has a very low stellar metallicity. For this work following Harmsen et al. 
(2017), we adopt a value of $[\mathrm{Fe} / \mathrm{H}] \sim-1.7$ dex at $\sim 30 \mathrm{kpc}$, which is the mean metallicity as reported by Sesar et al. (2011) and Xue et al. (2015). Within a heliocentric radius of $15 \mathrm{kpc}$, there is strong evidence of a fairly constant median metallicity (Carollo et al. 2007; de Jong et al. 2010; Xue et al. 2015). Even within the solar neighbourhood, the metallicity of the kinematically hot halo stars is consistent with a flat metallicity gradient of accreted stars (Beers et al. 2000). Further out beyond $15 \mathrm{kpc}$, there seems to be a tension between the results of Sesar et al. (2011) who find no metallicity gradient and de Jong et al. (2010) and Xue et al. (2015) who find a metallicity gradient. Nevertheless, for our purposes, the total accreted stellar metallicity cannot be larger than -1.4 dex. This indicates that the MW accreted stellar component was built from a number of smaller progenitors (low frac $\left._{\text {Dom }}\right)$ and the most massive satellite to contribute to the accreted stellar component had a stellar mass much smaller than the SMC (less than log $\mathrm{M}_{\mathrm{Dom} *} \sim 7.5$ ). The low metallicity of the total accreted stellar component also constrains the time of accretion to greater than $z>1$ (see Figure 14).

We note that the metallicities do not include significant contributions from the Sagittarius stream. Indeed, given metallicity and mass estimates for Sagittarius and its stream of $[\mathrm{Fe} / \mathrm{H}] \sim-0.7$ (Hyde et al. 2015) and $\mathrm{M}_{*}>10^{8} M_{\odot}$ (Niederste-Ostholt et al. 2010) respectively, Sagittarius will be the dominant progenitor of the Milky Way's stellar halo when it disrupts completely.

\section{M31}

The stellar halo of M31, the closest MW-like mass spiral galaxy has been well studied over the last decade. It would be interesting to test if the simple modelling adopted in this work can explain the detailed measurements of the accreted stellar component of M31.

M31 has a fairly large stellar halo compared to the MW. The stellar halo mass of M31 outside $27 \mathrm{kpc}$ is estimated to be $\sim 1.1 \times 10^{10} \mathrm{M}_{\odot}$ (Ibata et al. 2014). Following Harmsen et al. (2017), we assume the total accreted stellar mass of M31 is $\mathrm{M}_{\mathrm{Acc}, \mathrm{M} 31} \sim 1.5 \pm 0.5 \times 10^{10} \mathrm{M}_{\odot}$. Gilbert et al. (2014) estimated a metallicity gradient along the minor axis between 10 and $90 \mathrm{kpc}$ as $-0.01 \mathrm{dex} \mathrm{kpc}^{-1}$. The metallicity of the stellar halo of M31 at at $30 \mathrm{kpc}$ is estimated to be $[\mathrm{Fe} / \mathrm{H}] \sim-0.65$ (Gilbert et al. 2014) assuming a 10 Gyrs population. Extrapolating the metallicity gradient, the metallicity of the total accreted stellar component of M31 is estimated to be $[\mathrm{Fe} / \mathrm{H}] \sim-0.3$, which suggests that the stellar mass of the most dominant progenitor was $\log \mathrm{M}_{\mathrm{Dom} *} \sim 10.0$. Moreover, the reasonably steep metallicity gradient along the minor axis given M31's accreted stellar metallicity suggests that the dominant progenitor was accreted within a lookback time of 4-5 gigayears (see Figure 14).

A prominent feature of the inner spheroidal halo is the presence of a giant stellar stream (GSS) (Ibata et al. 2001). It would be interesting to speculate whether the progenitor of the GSS is the same as the dominant progenitor we predict through our models. Over the last decade, through a combination of TRGB line-of-sight distances (McConnachie et al. 2003) and radial velocity measurements of the tidal debris of the GSS (Ibata et al. 2004; Gilbert et al. 2007, 2009), the possible orbit of the GSS progenitor has been identified, showing that it fell almost straight into M31 from behind on a highly radial orbit. Moreover, the combination of velocity measurements, population studies and dynamical models have been able to demonstrate that a number of the tidal features of the inner spheroidal halo like the "West Shelf" and the "North-East Shelf" are caustics derived from the leading trail of the progenitor of the GSS (Font et al. 2008; Fardal et al. 2006, 2007, 2008; Mori \& Rich 2008; Fardal et al. 2013; Sadoun et al. 2014). While the dynamical models differ in various aspects, they generally agree on the fact that the progenitors initial stellar mass was in the range $1-5 \times 10^{9} M_{\odot}$. Detailed population studies of the GSS indicate that its star formation history is very similar to that of the inner spheroidal halo (Brown et al. 2006). Moreover, the star formation of the progenitor of the GSS shut off around 5 Gyrs (Brown et al. 2006; Bernard et al. 2015). This is commonly interpreted as the time the GSS progenitor entered the main halo of M31. The similarities between the predictions of our model and a possible progenitor of the GSS are encouraging; we postpone a more comprehensive analysis to a future publication.

\section{Cen $A(N G C 5128)$}

Cen A is the nearest well studied giant elliptical galaxy with detailed HST observations of its stellar halo extending out to large distances $\left(25 \mathrm{R}_{\mathrm{e}} \sim 150 \mathrm{kpc}\right.$; Rejkuba et al. 2014). A metal rich component dominates the stellar halo out to the most radially extended fields $([\mathrm{Fe} / \mathrm{H}]$ $>-1$ dex). It also exhibits a clear metallicity gradient $(\Delta[\mathrm{Fe} / \mathrm{H}] / \Delta \mathrm{R} \simeq-0.0054 \mathrm{dex} / \mathrm{kpc})$ out to $25 \mathrm{R}_{\mathrm{e}}$.

As is typical of elliptical galaxies, it is difficult to estimate its accreted stellar mass, owing to our inability to distinguish the in situ from the accreted stellar component. Assuming that the stellar populations beyond $60 \mathrm{kpc}$ are predominately accreted material, we can obtain estimates of the mass of the most dominant progenitor as well as the total accreted stellar mass.

Extrapolating the outer metallicity gradient of Cen A (at $60 \mathrm{kpc}$ ), we obtain a total accreted stellar metallicity of $[\mathrm{M} / \mathrm{H}] \sim-0.05$ dex, following Fig. 18 (or $[\mathrm{Fe} / \mathrm{H}] \sim-0.31$ dex, taking into consideration an assumed alpha enhancement of $[\alpha / \mathrm{Fe}]=0.3)$. The uncertainty in the total accreted stellar metallicity is around 0.20 dex. Using the metallicity-stellar mass relationship (Gallazzi et al. 2005), this suggests that Cen A accreted a massive progenitor with $\log \mathrm{M}_{\text {Dom * }} \sim 10.0 \pm 0.3$.

Integrating the outer stellar light of Cen $\mathrm{A}$, we find a total stellar mass of $\sim 5 \times 10^{9} \mathrm{M}_{\odot}$ between 60 and $150 \mathrm{kpc}$ along the major axis. Using model of the accreted stellar component of NGC 5128-equivalent galaxies in the Illustris simulations (similar to Section 6), we find that the total accreted stellar mass is 8 times the aperture measurement between 60 and $150 \mathrm{kpc}$, albeit with a larger 0.3 dex scatter. This suggests that the accreted stellar mass of NGC5128 is $\log \mathrm{M}_{\mathrm{acc}} \sim 10.6 \pm 0.3$. While extrapolating the aperture stellar mass provides an upper limit for the accreted stellar mass, the mass of the dominant progenitor provides a lower limit. This is the first time that the accreted stellar mass of an elliptical galaxy has been constrained to our knowledge. It is difficult to determine constraints on the time of accretion based on the density and metallicity gradients as we 
do not have observations of a larger sample of Cen A-type galaxies to compare with.

\subsection{Crafting a way forward}

Although we have measurements of the galaxy metallicitystellar mass relationship over a large range in stellar mass (Gallazzi et al. 2005; Kirby et al. 2013), a particular lacuna exists around existing measurements for progenitors of MWlike mass galaxies $\left(8.5<\log M_{*}<10.5\right)$. More effort needs to be invested in constraining the stellar metallicities of a volume limited sample of LMC/SMC like objects.

The evolution in metallicity-stellar mass relationship could be inferred using the accreted stellar metallicities and masses of a bigger volume-limited sample of MW-like galaxies. Yet, an independent measure of the evolution of the stellar metallicities as a function of stellar mass at high redshift $(z \sim 2)$ would be very valuable, and would considerably strengthen the robustness of analyses of stellar halos. Larger upcoming optical surveys like VANDELS (a deep VIMOS survey of CANDELS UDS and CDFS fields; http://vandels.inaf.it) or LEGA-C (van der Wel et al. 2016) will target star forming galaxies at a range of redshifts up to $z>2.5$ and shed greater light on the evolution of the galaxy metallicity-stellar mass relationship.

Although we have used the large number statistics of the Illustris simulations to our advantage, at some point, we are limited by its resolution. Higher resolution simulations are needed to confirm these results as well as better calibrate how we can extrapolate from aperture measurements of the stellar halo to the global measurements of the accreted stellar component of galaxies.

We did not consider in this work the information encoded in the velocity distribution of the accreted stellar component. Using dissipationless particle-tagging simulations, Amorisco (2017a) suggests that the angular momentum of the most massive accretion events is consumed and diluted by dynamical friction, resulting in almost non-rotating contributions with a strong radial bias. On the other hand, material deposited by low-mass satellites retain a significant amount of ordered rotations in the outer parts of galaxies. Taking these results at face value, this suggests that the velocities of outer halo stars may be able to inform us about smaller accretion events; larger progenitors may be more affected by the disk potential and dissipation (see, e.g., Gómez et al. 2017). It will be important to explore kinematic signatures of accretion for a wider range of halo masses, and using simulations with baryons, incorporating both dissipation and the potential from the disk of the main galaxy.

While the bulk properties of the accreted stellar component are shaped by the most dominant progenitor, the presence of substructure in the stellar halo can further inform us about the properties of smaller and more recent accretion events (e.g., Johnston et al. 2008). Quantifying faint substructure requires wider area coverage at deep surface brightness limits than characterization of the bulk stellar halo, and will benefit from surveys with Hyper Suprime-Cam (Miyazaki et al. 2012), LSST (Large Synoptic Survey Tele- scope LSST Science Collaboration et al. 2009) and WFIRST (Wide Field Infrared Survey Telescope) ${ }^{2}$.

Constraints on star formation histories may be of particular value in enriching our view of galactic accretion histories. While intermediate age AGB stars are bright and may be useful near-term probes for intermediate stellar populations for nearby galaxies (Greggio et al. 2014), most probes of population age will require the advent of larger aperture telescopes. The presence of particular old stellar populations (like RR-Lyrae or BHB stars) contain further information about older accretion events (Bell et al. 2010; Deason et al. 2012). Alpha-abundances and their radial trends contain information about star formation timescales, but require deep spectroscopy for accurate measurement.

\section{CONCLUSIONS}

In this paper, we demonstrate using the Illustris simulations the existence of a generalized accreted metallicity-stellar mass relationship, extending over three orders in magnitude in accreted stellar mass. This relationship is similar to the galaxy metallicity-stellar mass relationship, and is offset lower by $\sim 0.3$ dex.

The accreted metallicity-stellar mass relationship arises because the metallicity of the stellar halo is driven by the dominant progenitor. More massive satellites have a higher metallicity due to the galaxy metallicity-stellar mass relationship. Moreover, the dominant progenitor contributes the bulk of the stellar mass to the accreted stellar component. Galaxies with a similar DM halo mass range occupy a steeper locus across the accreted metallicity-stellar mass relationship.

We find that the scatter in accreted stellar metallicity at a fixed accreted stellar mass encodes information information about the mass of the dominant progenitor. On the other hand, we find that observable quantities containing spatial information (e.g. half-mass radius, density profile, metallicity gradients) of the accreted stellar halo encodes information about the time of accretion of the dominant progenitor.

With the goal of using these metrics of galaxy merger history for nearby galaxies, we explore possible methods for connecting minor axis resolved stellar population observations with model predictions. We can reconstruct the median total accreted stellar metallicity of a galaxy to an accuracy of 0.1 dex using a measurement of the accreted stellar metallicity along the minor axis of edge-on systems coupled with the metallicity gradients at large galacto-centric distances. "Aperture" measurements of the stellar halo mass can be extrapolated to the total accreted stellar mass to within an accuracy of 0.15 dex, with further increases in accuracy possible by using information encoded in the accreted density profile. We conclude that minor axis observations of resolved populations in stellar halos indeed hold considerable promise for empirically measuring the mass and timing of the dominant accretion event.

We explore the consequences of our models on the accretion histories of the MW, M31 and NGC 5128. We find the

\footnotetext{
2 https://wfirst.gsfc.nasa.gov/science/sdt_public/

WFIRST-AFTA_SDT_Report_150310_Final.pdf
} 
most massive satellite to contribute to the accreted stellar component of the MW (excepting Sagittarius) had a stellar mass much smaller than the SMC (less than log $\mathrm{M}_{\text {Dom * }} \sim$ 7.5 ) and was accreted earlier that $z \sim 1$. On the other hand, our models predict that M31 accreted a satellite of stellar mass larger than log $\mathrm{M}_{\mathrm{Dom} *} \sim 9.7 \pm 0.2$ about 4-5 gigayears ago. We also constrain the stellar mass of the dominant progenitor and the accreted stellar mass of NGC 5128 as $\log \mathrm{M}_{\text {Dom * }} \sim 10 \pm 0.3$ and $\log \mathrm{M}_{\mathrm{acc}} \sim 10.6 \pm 0.3$ respectively; to our knowledge this is the first estimate to have been made of the accreted stellar mass of NGC 5128 .

\section{ACKNOWLEDGEMENTS}

We thank Alis Deason for providing the properties of her modelled stellar halos in electronic form. We acknowledge useful discussions with Antonela Monachesi, Kohei Hattori, Sarah Loebman, Monica Valluri, Ian Roederer and Adam Smercina. We also would like to thank the Illustris simulations team, in particular Annalisa Pillepich, Vincent Rodrigues-Gomez and Dylan Nelson.

\section{REFERENCES}

Abadi M. G., Navarro J. F., Steinmetz M., 2006, MNRAS, 365,747

Amorisco N. C., 2017a, MNRAS, 464, 2882

Amorisco N. C., 2017b, ArXiv e-prints

Beers T. C., Chiba M., Yoshii Y., Platais I., et al., 2000, AJ, 119, 2866

Bell E. F., Monachesi A., Harmsen B., de Jong R. S., et al., 2017, ApJL, 837, L8

Bell E. F., Xue X. X., Rix H.-W., Ruhland C., Hogg D. W., 2010, AJ, 140, 1850

Bell E. F., Zucker D. B., Belokurov V., Sharma S., et al., 2008, ApJ, 680, 295

Bernard E. J., Ferguson A. M. N., Richardson J. C., Irwin M. J., et al., 2015, MNRAS, 446, 2789

Bland-Hawthorn J., Gerhard O., 2016, Annual Review of Astronomy \& Astrophysics, 54, 529

Brown T. M., Smith E., Ferguson H. C., Rich R. M., Guhathakurta P., Renzini A., Sweigart A. V., Kimble R. A., 2006, ApJ, 652, 323

Bullock J. S., Johnston K. V., 2005, ApJ, 635, 931

Carlin J. L., Sand D. J., Price P., Willman B., Karunakaran A., Spekkens K., Bell E. F., Brodie J. P., Crnojević D., Forbes D. A., Hargis J., Kirby E., Lupton R., Peter A. H. G., Romanowsky A. J., Strader J., 2016, ApJL, 828, L5

Carollo D., Beers T. C., Lee Y. S., Chiba M., et al., 2007, Nature, 450, 1020

Cook B. A., Conroy C., Pillepich A., Rodriguez-Gomez V., et al., 2016, ApJ, 833, 158

Cooper A. P., Cole S., Frenk C. S., White S. D. M., et al., 2010, MNRAS, 406, 744

Cooper A. P., D'Souza R., Kauffmann G., Wang J., et al., 2013, MNRAS, 434, 3348

Cooper A. P., Parry O. H., Lowing B., Cole S., et al., 2015, MNRAS, 454, 3185
Crnojević D., Sand D. J., Spekkens K., Caldwell N., et al., 2016, ApJ, 823, 19

Davis M., Efstathiou G., Frenk C. S., White S. D. M., 1985, ApJ, 292, 371

de Jong J. T. A., Yanny B., Rix H.-W., Dolphin A. E., Martin N. F., Beers T. C., 2010, ApJ, 714, 663

Deason A. J., Belokurov V., Evans N. W., Koposov S. E., Cooke R. J., Peñarrubia J., Laporte C. F. P., Fellhauer M., Walker M. G., Olszewski E. W., 2012, MNRAS, 425, 2840

Deason A. J., Mao Y.-Y., Wechsler R. H., 2016, ApJ, 821, 5

Dolag K., Borgani S., Murante G., Springel V., 2009, MNRAS, 399, 497

Duc P.-A., Cuillandre J.-C., Karabal E., Cappellari M., et al., 2015, MNRAS, 446, 120

Durrell P. R., Harris W. E., Pritchet C. J., 2001, ApJ, 121, 2557

Durrell P. R., Harris W. E., Pritchet C. J., 2004, ApJ, 128, 260

Fakhouri O., Ma C.-P., Boylan-Kolchin M., 2010, MNRAS, 406, 2267

Fardal M. A., Babul A., Geehan J. J., Guhathakurta P., 2006, MNRAS, 366, 1012

Fardal M. A., Babul A., Guhathakurta P., Gilbert K. M., Dodge C., 2008, ApJL, 682, L33

Fardal M. A., Guhathakurta P., Babul A., McConnachie A. W., 2007, MNRAS, 380, 15

Fardal M. A., Weinberg M. D., Babul A., Irwin M. J., Guhathakurta P., Gilbert K. M., Ferguson A. M. N., Ibata R. A., Lewis G. F., Tanvir N. R., Huxor A. P., 2013, MNRAS, 434, 2779

Ferguson A. M. N., Irwin M. J., Ibata R. A., Lewis G. F., et al., 2002, ApJ, 124, 1452

Font A. S., Johnston K. V., Bullock J. S., Robertson B. E., 2006, ApJ, 646, 886

Font A. S., Johnston K. V., Ferguson A. M. N., Bullock J. S., Robertson B. E., Tumlinson J., Guhathakurta P., 2008, ApJ, 673, 215

Font A. S., McCarthy I. G., Crain R. A., Theuns T., et al., 2011, MNRAS, 416, 2802

Gallazzi A., Bell E. F., Zibetti S., Brinchmann J., et al., 2014, ApJ, 788, 72

Gallazzi A., Charlot S., Brinchmann J., White S. D. M., et al., 2005, MNRAS, 362, 41

Garrison-Kimmel S., Boylan-Kolchin M., Bullock J. S., Lee K., 2014, MNRAS, 438, 2578

Genel S., Vogelsberger M., Springel V., Sijacki D., et al., 2014, MNRAS, 445, 175

Gilbert K. M., Fardal M., Kalirai J. S., Guhathakurta P., Geha M. C., Isler J., Majewski S. R., Ostheimer J. C., Patterson R. J., Reitzel D. B., Kirby E., Cooper M. C., 2007, ApJ, 668, 245

Gilbert K. M., Guhathakurta P., Beaton R. L., Bullock J., et al., 2012, ApJ, 760, 76

Gilbert K. M., Guhathakurta P., Kollipara P., Beaton R. L., Geha M. C., Kalirai J. S., Kirby E. N., Majewski S. R., Patterson R. J., 2009, ApJ, 705, 1275

Gilbert K. M., Kalirai J. S., Guhathakurta P., Beaton R. L., et al., 2014, ApJ, 796, 76

Gómez F. A., Grand R. J. J., Monachesi A., White S. D. M., et al., 2017, ArXiv e-prints 
Greggio L., Rejkuba M., Gonzalez O. A., Arnaboldi M., Iodice E., Irwin M., Neeser M. J., Emerson J., 2014, A\&A, 562, A73

Halliday C., Daddi E., Cimatti A., Kurk J., et al., 2008, A\&A, 479, 417

Harmsen B., Monachesi A., Bell E. F., de Jong R. S., et al., 2017, MNRAS, 466, 1491

Hirschmann M., Naab T., Davé R., Oppenheimer B. D., et al., 2013, MNRAS, 436, 2929

Hirschmann M., Naab T., Ostriker J. P., Forbes D. A., et al., 2015, MNRAS, 449, 528

Hyde E. A., Keller S., Zucker D. B., Ibata R., Siebert A., Lewis G. F., Penarrubia J., Irwin M., Gilmore G., Lane R. R., Koch A., Conn A. R., Diakogiannis F. I., Martell S., 2015, ApJ, 805, 189

Ibata R., Chapman S., Ferguson A. M. N., Irwin M., Lewis G., McConnachie A., 2004, MNRAS, 351, 117

Ibata R., Irwin M., Lewis G., Ferguson A. M. N., Tanvir N., 2001, Nature, 412, 49

Ibata R. A., Lewis G. F., McConnachie A. W., Martin N. F., et al., 2014, ApJ, 780, 128

Irwin M. J., Ferguson A. M. N., Ibata R. A., Lewis G. F., Tanvir N. R., 2005, ApJL, 628, L105

Johnston K. V., Bullock J. S., Sharma S., Font A., et al., 2008, ApJ, 689, 936

Kalirai J. S., Gilbert K. M., Guhathakurta P., Majewski S. R., Ostheimer J. C., Rich R. M., Cooper M. C., Reitzel D. B., Patterson R. J., 2006, ApJ, 648, 389

Kirby E. N., Cohen J. G., Guhathakurta P., Cheng L., et al., 2013, ApJ, 779, 102

LSST Science Collaboration Abell P. A., Allison J., Anderson S. F., et al., 2009, ArXiv e-prints

Ma X., Hopkins P. F., Faucher-Giguère C.-A., Zolman N., et al., 2016, MNRAS, 456, 2140

McConnachie A. W., Irwin M. J., Ibata R. A., Ferguson A. M. N., Lewis G. F., Tanvir N., 2003, MNRAS, 343, 1335

Merritt A., van Dokkum P., Abraham R., Zhang J., 2016, ApJ, 830, 62

Miyazaki S., Komiyama Y., Nakaya H., Kamata Y., et al., 2012, in Ground-based and Airborne Instrumentation for Astronomy IV Vol. 8446 of Proceedings of SPIE, Hyper Suprime-Cam. p. $84460 \mathrm{Z}$

Monachesi A., Bell E. F., Radburn-Smith D. J., Bailin J., et al., 2016, MNRAS, 457, 1419

Monachesi A., Bell E. F., Radburn-Smith D. J., Vlajić M., et al., 2013, ApJ, 766, 106

Monachesi A., Gómez F. A., Grand R. J. J., Kauffmann G., et al., 2016, MNRAS, 459, L46

Mori M., Rich R. M., 2008, ApJL, 674, L77

Mouhcine M., 2006, ApJ, 652, 277

Mouhcine M., Ferguson H. C., Rich R. M., Brown T. M., et al., 2005, ApJ, 633, 821

Mouhcine M., Ibata R., Rejkuba M., 2010, ApJL, 714, L12

Mouhcine M., Rich R. M., Ferguson H. C., Brown T. M., et al., 2005, ApJ, 633, 828

Mould J., Kristian J., 1986, ApJ, 305, 591

Navarro J. F., Frenk C. S., White S. D. M., 1997, ApJ, 490, 493

Nelson D., Pillepich A., Genel S., Vogelsberger M., et al., 2015, Astronomy and Computing, 13, 12

Niederste-Ostholt M., Belokurov V., Evans N. W.,
Peñarrubia J., 2010, ApJ, 712, 516

Pillepich A., Madau P., Mayer L., 2015, ApJ, 799, 184

Pillepich A., Vogelsberger M., Deason A., Rodriguez-

Gomez V., et al., 2014, MNRAS, 444, 237

Purcell C. W., Bullock J. S., Zentner A. R., 2007, ApJ, 666, 20

Purcell C. W., Bullock J. S., Zentner A. R., 2008, MNRAS, 391, 550

Radburn-Smith D. J., de Jong R. S., Seth A. C., Bailin J., et al., 2011, ApJS, 195, 18

Reitzel D. B., Guhathakurta P., Gould A., 1998, ApJ, 116, 707

Rejkuba M., Harris W. E., Greggio L., Harris G. L. H., 2011, A\&A, 526, A123

Rejkuba M., Harris W. E., Greggio L., Harris G. L. H., et al., 2014, ApJL, 791, L2

Renda A., Gibson B. K., Mouhcine M., Ibata R. A., et al., 2005, MNRAS, 363, L16

Rix S. A., Pettini M., Leitherer C., Bresolin F., et al., 2004, ApJ, 615, 98

Robertson B., Bullock J. S., Font A. S., Johnston K. V., et al., 2005, ApJ, 632, 872

Rodriguez-Gomez V., Genel S., Vogelsberger M., Sijacki D., et al., 2015, MNRAS, 449, 49

Rodriguez-Gomez V., Pillepich A., Sales L. V., Genel S., et al., 2016, MNRAS, 458, 2371

Sadoun R., Mohayaee R., Colin J., 2014, MNRAS, 442, 160

Saglia R. P., Fabricius M., Bender R., Montalto M., Lee C.H., Riffeser A., Seitz S., Morganti L., Gerhard O., Hopp U., 2010, A\&A, 509, A61

Schaye J., Crain R. A., Bower R. G., Furlong M., et al., 2015, MNRAS, 446, 521

Sesar B., Jurić M., Ivezić Ž., 2011, ApJ, 731, 4

Snyder G. F., Torrey P., Lotz J. M., Genel S., et al., 2015, MNRAS, 454, 1886

Sommariva V., Mannucci F., Cresci G., Maiolino R., et al., 2012, A\&A, 539, A136

Springel V., 2010, Annual Review of Astronomy \& Astrophysics, 48, 391

Springel V., White S. D. M., Tormen G., Kauffmann G., 2001, MNRAS, 328, 726

Tissera P. B., Scannapieco C., Beers T. C., Carollo D., 2013, MNRAS, 432, 3391

Trujillo I., Fliri J., 2016, ApJ, 823, 123

van der Wel A., Noeske K., Bezanson R., Pacifici C., et al., 2016, ApJS, 223, 29

van Dokkum P. G., Abraham R., Merritt A., 2014, ApJL, 782, L24

Vogelsberger M., Genel S., Sijacki D., Torrey P., et al., 2013, MNRAS, 436, 3031

Vogelsberger M., Genel S., Springel V., Torrey P., et al., 2014a, MNRAS, 444, 1518

Vogelsberger M., Genel S., Springel V., Torrey P., et al., 2014b, Nature, 509, 177

Woo J., Courteau S., Dekel A., 2008, MNRAS, 390, 1453

Xue X.-X., Rix H.-W., Ma Z., Morrison H., et al., 2015, ApJ, 809, 144

Zolotov A., Willman B., Brooks A. M., Governato F., et al., 2009, ApJ, 702, 1058 